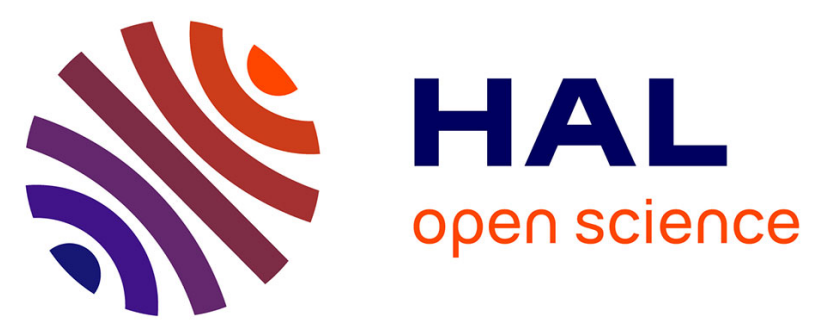

\title{
Nonstationarity of strong collisionless quasiperpendicular shocks: Theory and full particle numerical simulations
}

Vladimir Krasnoselskikh, Bertrand Lembège, P. Savoini, V. V. Lobzin

\section{To cite this version:}

Vladimir Krasnoselskikh, Bertrand Lembège, P. Savoini, V. V. Lobzin. Nonstationarity of strong collisionless quasiperpendicular shocks: Theory and full particle numerical simulations. Physics of Plasmas, 2002, 9 (4), pp.1192-1209. 10.1063/1.1457465 . insu-02928445

HAL Id: insu-02928445

https://hal-insu.archives-ouvertes.fr/insu-02928445

Submitted on 2 Sep 2020

HAL is a multi-disciplinary open access archive for the deposit and dissemination of scientific research documents, whether they are published or not. The documents may come from teaching and research institutions in France or abroad, or from public or private research centers.
L'archive ouverte pluridisciplinaire HAL, est destinée au dépôt et à la diffusion de documents scientifiques de niveau recherche, publiés ou non, émanant des établissements d'enseignement et de recherche français ou étrangers, des laboratoires publics ou privés. 


\section{Nonstationarity of strong collisionless quasiperpendicular shocks: Theory and full particle numerical simulations}

Cite as: Physics of Plasmas 9, 1192 (2002); https://doi.org/10.1063/1.1457465

Submitted: 05 June 2001 . Accepted: 09 January 2002 . Published Online: 21 March 2002

V. V. Krasnoselskikh, B. Lembège, P. Savoini, and V. V. Lobzin

\section{ARTICLES YOU MAY BE INTERESTED IN}

Shock front instability associated with reflected ions at the perpendicular shock

Physics of Plasmas 14, 012108 (2007); https://doi.org/10.1063/1.2435317

Whistler waves, core ion heating, and nonstationarity in oblique collisionless shocks

Physics of Plasmas 14, 072103 (2007); https://doi.org/10.1063/1.2748391

Nonstationarity of a two-dimensional quasiperpendicular supercritical collisionless shock by self-reformation

Physics of Fluids B: Plasma Physics 4, 3533 (1992); https://doi.org/10.1063/1.860361

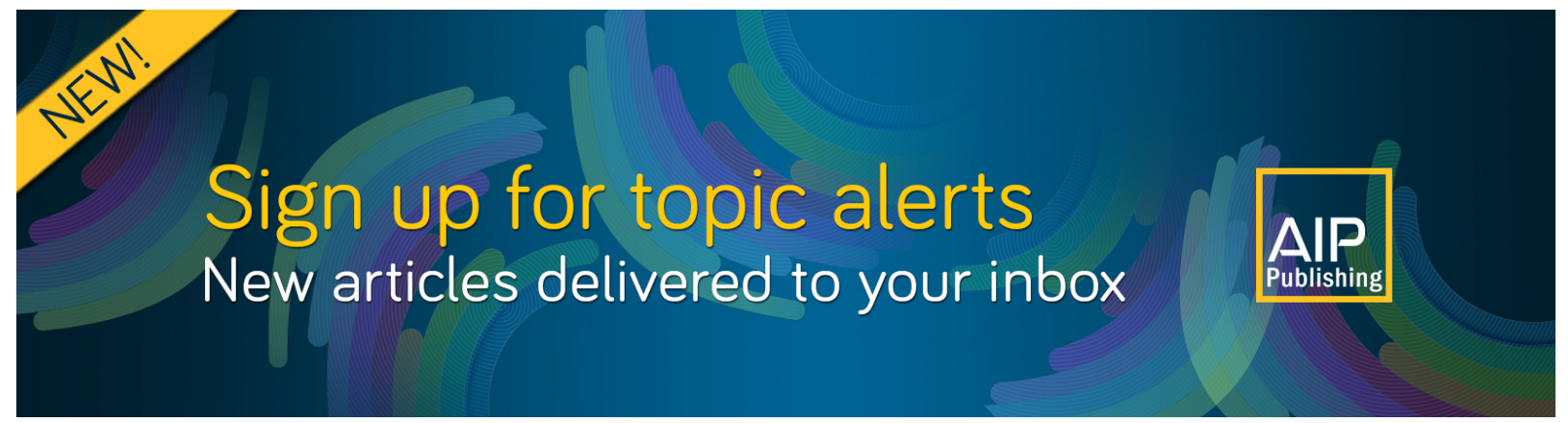




\title{
Nonstationarity of strong collisionless quasiperpendicular shocks: Theory and full particle numerical simulations
}

\author{
V. V. Krasnoselskikha) \\ LPCE/CNRS, Orléans, France \\ B. Lembège and P. Savoini \\ CETP/CNRS, Velizy, France \\ V. V. Lobzin ${ }^{\text {b) }}$ \\ LPCE/CNRS, Orléans, France
}

(Received 5 June 2001; accepted 9 January 2002)

\begin{abstract}
Whistler waves are an intrinsic feature of the oblique quasiperpendicular collisionless shock waves. For supercritical shock waves, the ramp region, where an abrupt increase of the magnetic field occurs, can be treated as a nonlinear whistler wave of large amplitude. In addition, oblique shock waves can possess a linear whistler precursor. There exist two critical Mach numbers related to the whistler components of the shock wave, the first is known as a whistler critical Mach number and the second can be referred to as a nonlinear whistler critical Mach number. When the whistler critical Much number is exceeded, a stationary linear wave train cannot stand ahead of the ramp. Above the nonlinear whistler critical Mach number, the stationary nonlinear wave train cannot exist anymore within the shock front. This happens when the nonlinear wave steepening cannot be balanced by the effects of the dispersion and dissipation. In this case nonlinear wave train becomes unstable with respect to overturning. In the present paper it is shown that the nonlinear whistler critical Mach number corresponds to the transition between stationary and nonstationary dynamical behavior of the shock wave. The results of the computer simulations making use of the 1D full particle electromagnetic code demonstrate that the transition to the nonstationarity of the shock front structure is always accompanied by the disappearance of the whistler wave train within the shock front. Using the two-fluid MHD equations, the structure of nonlinear whistler waves in plasmas with finite beta is investigated and the nonlinear whistler critical Mach number is determined. It is suggested a new more general proof of the criteria for small amplitude linear precursor or wake wave trains to exist. (C) 2002 American Institute of Physics. [DOI: 10.1063/1.1457465]
\end{abstract}

\section{INTRODUCTION}

Shock waves in plasmas as well as in gases and other media are usually considered to be nonlinear waves that cause changes of state of the media and are stationary in some reference frames. Indeed, numerous theoretical papers are devoted to finding stationary solutions to the set of equations describing the plasma dynamics (for a review, see, e.g., Refs. 1-3). However, in the very beginning of the collisionless shock physics, the hypothesis was suggested that high Mach number shocks can be nonstationary (see, e.g., Refs. 4, 5). As far as we know, the first unambiguous evidence of the shock wave nonstationarity was obtained by Morse et al. ${ }^{6}$ in the laboratory experiments with a plasma-wind-tunnel device. They revealed that in the fast mode Mach number range $M_{f} \simeq 4-8$ the shock wave oscillates with a frequency comparable to the upstream ion gyrofrequency.

In the early 1980s, in the response both to new observations of the Earth bow shock and a great progress both in computational sciences and computer hardware, the interest to the problem concerned is considerably increased. In the

\footnotetext{
${ }^{a)}$ Electronic mail: vkrasnos@cnrs-orleans.fr

${ }^{b}$ Permanent address: IZMIRAN, Troitsk, Moscow Region, Russia.
}

Earth bow shock, the low frequency oscillations of the ion flux were observed. ${ }^{7-9}$ Later similar phenomenon was also found in the uranium bow shock. ${ }^{10}$ These observations can easily be interpreted as a manifestation of the shock front nonstationarity. ${ }^{11,12}$ In addition, Leroy et al. ${ }^{13,14}$ modeled the dynamics of a perpendicular shocks with the use of a hybrid code. Simulations with the parameters typical for the Earth bow shock (in particular, for $M_{A}=8$ and $\beta_{e}=\beta_{i}=0.6$, where $M_{A}$ is the Alfvén Mach number, $\beta_{e, i}$ is the ratio of the thermal and magnetic pressures) showed that the shock structure varies with time, for example, the maximum value of the magnetic field exhibits temporal variations with a characteristic time of the order of ion gyroperiod, the magnitude of these variations being about of $20 \%$. The amplitude of the oscillations increases with the Mach number and decreasing $\beta_{i}$. The oscillations of the fraction of reflected ions, $\alpha$, are more pronounced than that of the magnetic field overshoot or the maximum value of electric potential. For $M_{A}=10$ and $\beta=0.1$ the ion reflection was bursty, $\alpha$ oscillating between $0 \%$ and $70 \%-75 \%$, although the magnetic field and electric potential overshoot can be considered as quasistationary on the average because their relative amplitudes of oscillations do not exceed $10 \%-15 \%$.

For the first time, modeling of high Mach number per- 
pendicular shocks $\left(M_{A}=22, \beta=0.1\right)$ was carried out by Quest. ${ }^{15} \mathrm{He}$ found that in the absence of the electron resistivity the ion reflection process is periodic, the stages with $100 \%$ ion reflection alternating with the stages of $100 \%$ ion transmission. As a result, a periodic shock front reformation was observed rather than a stationary structure. Quest ${ }^{16}$ extended these preliminary simulations to perform a systematic study of high Mach number perpendicular shocks. For $\beta$ $=0.1$ he revealed that the found previously ${ }^{14}$ tendency for a shock to become increasingly time-dependent as $M_{A}$ increases was also observed for $M_{A} \geqslant 10$ and resulted in the cyclical wave breaking for $M_{A} \geqslant 20$. In addition, for $\beta=1$ and $M_{A} \geqslant 10$ a non-trivial dependence of the shock front structure on the resistivity was found. Nonstationarity was observed both for low and high resistivity $\eta$ while for moderate $\eta$ the shocks were stationary up to $M_{A}=60$. In conclusion, Quest ${ }^{16}$ argued that a fundamental question concerned with a physical mechanism that controls the stability of the shocks has not been resolved yet.

In addition, it is worth noting that in numerical simulations the intrinsic shock front instability can be obscured by a number of unphysical effects such as an artificial dissipation, dispersion, and/or instability of the computational algorithm. ${ }^{11}$

Analyzing the major achievements of collisionless shock physics by the end of 1984 and reviewing the conceptual issues of the subject, Kennel et al. ${ }^{17}$ argued that the shock front nonstationarity does exist and they suggested to introduce the so-called third critical Mach number corresponding to the transition between the stationary shocks and nonstationary ones.

Krasnoselskikh ${ }^{18}$ and Galeev et al. ${ }^{19}$ suggested the theoretical models describing the shock front instability due to domination of the nonlinear effects over the dispersion and dissipation. This instability results in a gradient catastrophe within a finite time interval and nonstationarity of the shock wave. Later Galeev et al. ${ }^{20}$ showed that the nonlinear whistler wave train can be observed within the front of the quasiperpendicular shock wave under some conditions and they argued that the role of this wave train should be taken into account when analyzing the problem concerned. To confirm the hypothesis of shock front nonstationarity, Galeev et al. ${ }^{11}$ presented the results of analysis of the experimental data obtained onboard Prognoz-8 and Prognoz-10 for several crossings of the Earth bow shock. The manifestation of the shock front nonstationarity in the ion distribution function was also discussed. ${ }^{12}$ Later, the presence of the nonstationary whistler wave trains in the front of strong quasiperpendicular shock waves was also confirmed by direct observations of the Earth bow shock onboard Intershock-Prognoz-10 and AMPTE UK spacecrafts. ${ }^{21}$

Lembége $e t a l .^{22}$ also observed the cyclic reformation of the exactly perpendicular low-beta nonresistive shock waves in 1D full particle simulations, where the ratio of electron and ion masses was $\mu=m_{e} / m_{i}=0.01$. They argue that very high Mach numbers are not necessary for the reformation to exist; in these simulations the reformation was also observed for relatively low Mach numbers $M_{A}=2-3$ corresponding however to supercritical regimes. In the $2 \mathrm{D}$ full particle simulations with the mass ratio $\mu=0.024$, Lembége et al. ${ }^{23}$ found that the cyclic reformation of the front takes place also for oblique shock waves within the same Mach number range even when the finite resistivity effects due to crossfield-current instabilities are self-consistently included. The reformation cycle was found to be of the order of the mean ion gyroperiod measured in the shock ramp. Furthermore, the shock front appeared to be rippled rather than uniform. However, the discussion of these two-dimensional effects is beyond the scope of the present paper.

The present paper is organized as follows: In Sec. II, the model suggested in Ref. 19 is briefly outlined. We believe that this model describes not only shock front formation for moderate Mach numbers but the nonstationary reformation of strong shocks as well. Several aspects of the model are developed in further detail and more rigorously. In particular, we analyze a model equation describing the dynamics of the shock wave front with large gradients, when a characteristic length of the plasma flow within the front is typical for whistler waves. This analysis shows that the dispersion of whistler waves is not sufficient to prevent the breaking of strong disturbances. On the basis of this analysis, we put forward an argumentation that a nonlinear whistler critical Mach number, above which the nonlinear whistler wave train cannot stand within the shock front, is approximately equal to the critical Mach number corresponding to the transition from stationary shock waves to nonstationary ones. In Sec. III we present the results of the computer simulations making use of the 1D full particle electromagnetic code. In order to obtain more reliable results concerned with the role of whistler waves in the shock front nonstationarity, we take a smaller ratio of electron and ion masses, $\mu=0.005$, than used previously. ${ }^{22,23}$ To distinguish between the transient processes during formation of a stationary shock and the intrinsic nonstationarity of strong shocks, in the present simulations the total run time covers about 4 ion gyroperiods calculated with the use of upstream magnetic field (previously ${ }^{22,23}$ the duration of the runs did not exceed 0.5-1 ion gyroperiods). The results obtained demonstrate that the transition to the nonstationarity is always accompanied by the disappearance of the phase-standing whistler wave train within the shock front. Some auxiliary facts and results are presented in the Appendices. In particular, Appendix B contains a new more general proof of the criteria for smallamplitude precursor or wake wave trains to exist. In Appendix $C$ the structure of nonlinear whistlers in plasmas with finite $\beta$ and adiabatic equation of state for electrons is investigated using two-fluid MHD equations.

\section{GRADIENT CATASTROPHES AND INSTABILITY OF STATIONARY SHOCK FRONT STRUCTURE}

\section{A. A multistage process of the formation of a strong shock wave}

As opposed to hydrodynamic shocks, shock waves in collisionless plasmas usually have much more complicated structure. For example, a typical magnetic field profile for a quasiperpendicular supercritical shock is shown in Fig. 1 taken from Ref. 24. It consists of a foot or pedestal, ramp, 


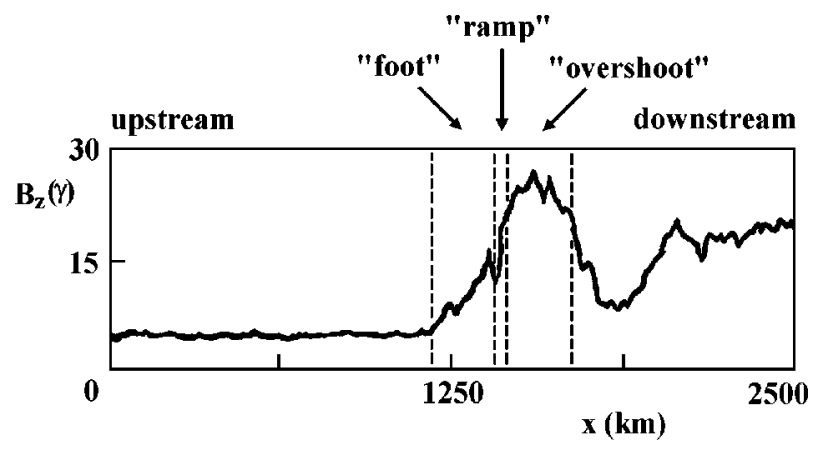

FIG. 1. The magnetic field profile for a typical supercritical quasiperpendicular shock wave obtained in numerical simulations (from Ref. 24 with kind permission from Kluwer Academic). The foot, ramp, and overshoot are indicated.

and overshoot-undershoot oscillations. This large-scale structure is closely related to the dynamics of reflected ions. A fraction of the incoming ions is reflected from the ramp, where the gradient of the magnetic field intensity is the largest, then they are magnetically deflected, accelerated by the flow electric field, and pass the ramp on the second encounter thereby forming the ring distribution in the overshootundershoot region. Assuming that the ions are specularly reflected from the ramp, the foot thickness can be estimated as $^{25}$

$$
L_{\text {foot }}=0.68 \frac{U_{0}}{\omega_{B i}} \sin \theta_{B n},
$$

where $U_{0}$ is the plasma velocity along the shock normal in the shock frame, $\omega_{B i}$ is the upstream ion gyrofrequency, and $\theta_{B n}$ is the angle between the shock normal and the upstream magnetic field. The distance between the overshoot and undershoot has the same order of magnitude. ${ }^{26}$ The ramp is significantly thinner, it follows from some observations that it can be of the order of several electron skin depths $c / \omega_{p e}$, where $c$ is the speed of light and $\omega_{p e}$ is the electron plasma frequency. The dispersion length $c \mid \cos \theta_{B n} / / \omega_{p i}$, where $\omega_{p i}$ is the ion plasma frequency, determines the "ramp" thickness for oblique subcritical shock waves. Although not explicitly presented in the large-scale structure, there is a number of other scales, which are closely related to the physical processes within the shock front. For instance, the Debye length electrostatic fluctuations due to the ion-sound instability are believed to provide an anomalous resistivity. The length of the order of several $c / \omega_{p e}$ is believed to be typical wavelength of whistler waves observed within the front and upstream. ${ }^{20,21}$ The thermal electron gyroradius, $\rho_{e}$ $=v_{T e} / \omega_{B e}$, where $v_{T e}$ is the electron thermal velocity and $\omega_{B e}$ is the electron gyrofrequency, determines the boundary between two regimes of electron heating, i.e., if the characteristic length of the plasma flow is much larger than $\rho_{e}$, the electron component obey an adiabatic equation of state, otherwise the nonadiabatic heating takes place.

Since some of the scales within this hierarchy differ from others by several orders of magnitude, the theoretical analysis of the shock formation is a very complicated problem. However, we can model different stages of the shock formation with the use of simplified sets of equations, where the main features of the stage considered are taken into account.

To begin with, let us consider the dispersion relation for fast magnetosonic waves. Indeed, there exists a multiform relationship between the structure of shock waves and the properties of the corresponding linear waves. ${ }^{1,2}$ In particular, when considering the evolution and structure of high Mach number shocks, the dispersion relation for fast magnetosonic waves is of particular importance. In the case of the cold plasma, the frequency dependence of the refraction index $\mathcal{N}=k c / \omega$ can be easily written in the analytic form (see, e.g., Ref. 27). However, this form of the dispersion relation is rather cumbersome and inconvenient to analyze. Instead of it, in the following we use an approximate relationship suggested by Krasnoselskikh et al. ${ }^{28}$

$$
\omega^{2}=\frac{\omega_{B e}^{2}}{1+\omega_{p e}^{2} / k^{2} c^{2}}\left(\mu+\frac{\cos ^{2} \theta}{1+\omega_{p e}^{2} / k^{2} c^{2}}\right),
$$

where $\omega$ and $k$ are the frequency and the wave number, respectively, $\theta$ is the angle between the wave vector and the magnetic field, and $\mu$ is the ratio of electron and ion masses, $\mu=m_{e} / m_{i}$. Although this equation is approximate, it describes all the relevant features of the exact dispersion relation. Indeed, for the waves propagating perpendicularly to the magnetic field $\left(\theta=90^{\circ}\right)$ from Eq. (1) we get

$$
\omega^{2}=\frac{k^{2} v_{A}^{2}}{1+k^{2} c^{2} / \omega_{p e}^{2}} .
$$

It is easily seen from Eq. (2) that the phase velocity of the waves is approximately equal to Alfvén velocity $v_{A}$ for $k c / \omega_{p e} \ll 1$ and decreases to zero as the wave number $k$ is increased and the frequency approaches the low hybrid frequency, $\omega \rightarrow\left(\omega_{B e} \omega_{B i}\right)^{1 / 2}$. For long waves, the characteristic dispersion length is $c / \omega_{p e}$. For oblique propagation, $\cos ^{2} \theta$ $\gg \mu$, the long waves have the same velocity $v_{A}$, but the phase velocity increases with the increase in $k$, and the characteristic dispersion length is equal to $c|\cos \theta| / \omega_{p i}$,

$$
\omega^{2} \simeq k^{2} v_{A}^{2}\left(1+\frac{k^{2} c^{2} \cos ^{2} \theta}{\omega_{p i}^{2}}\right) .
$$

The last equation describes also the low frequency whistlers with $\omega \ll \omega_{B e}|\cos \theta|$. Finally, for $k c / \omega_{p e} \gg 1$ the oblique waves are electrostatic with the frequency $\omega \simeq \omega_{B e}|\cos \theta|$ and small phase velocity.

Figure 2 shows the dependence of phase velocity $v_{\mathrm{ph}}$ $=\omega / \mathrm{k}$ upon the wave number in the case of oblique propagation, $\cos ^{2} \theta \gg \mu$. As it was noted above, the range of wave numbers can be split into three parts corresponding to (I) long waves, $k \ll \omega_{p i} / c|\cos \theta|$, (II) whistler waves with a characteristic wavelength of about $c / \omega_{p e}$, and (III) quasielectrostatic oscillations, respectively.

When considering a shock wave formation from a smooth initial disturbance of the plasma flow, we can imagine that a point representing the characteristic scale length of the disturbance moves along the dispersion curve shown in Fig. 2. Thereby the evolution of the disturbance resulting in 


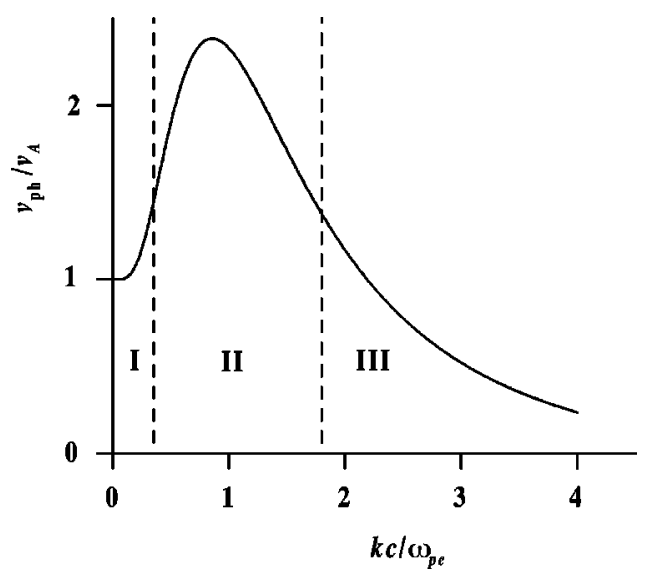

FIG. 2. The dependence of phase velocity of the oblique fast magnetosonic waves on the wave number.

the shock formation can be considered as a three-stage process, each stage corresponding to a particular part of the dispersion relation.

In this paper we consider the problem of the formation of oblique shocks such that $\cos ^{2} \theta \gg \mu$.

\section{B. Large scale phenomena and evolution of the MHD type system}

Consider a one-dimensional plasma flow such that initially it is plane-polarized and consists of three regions, namely, two regions of approximately steady flow with considerably different velocities and the third region between the two. Suppose further that the gradients in the third region are small enough, i.e., a characteristic length of the disturbance corresponds to the region I on the dispersion curve (see Fig. 2), i.e., this length is much greater than the characteristic lengths related to both dispersion and dissipation. Choose a reference frame such that the only nonvanishing components of the initial disturbance of the magnetic field and plasma velocity are $B_{x, y}$ and $V_{x, y}$, respectively. Such a flow is governed by the well-known equations of magnetohydrodynamics of an ideal medium (see, e.g., Refs. 29, 30),

$$
\begin{aligned}
& \frac{\partial \rho}{\partial t}+\frac{\partial}{\partial x}\left(\rho V_{x}\right)=0, \\
& \frac{\partial V_{x}}{\partial t}+V_{x} \frac{\partial V_{x}}{\partial x}=-\frac{c_{s}^{2}}{\rho} \frac{\partial \rho}{\partial x}-\frac{B_{y}}{4 \pi \rho} \frac{\partial B_{y}}{\partial x}, \\
& \frac{\partial V_{y}}{\partial t}+V_{x} \frac{\partial V_{y}}{\partial x}=\frac{B_{x}}{4 \pi \rho} \frac{\partial B_{y}}{\partial x}, \\
& \frac{\partial B_{y}}{\partial t}+V_{x} \frac{\partial B_{y}}{\partial x}=B_{x} \frac{\partial V_{y}}{\partial x}-B_{y} \frac{\partial V_{x}}{\partial x},
\end{aligned}
$$

where $\rho$ is the plasma density, $c_{s}$ is the sound velocity, and $B_{x}=$ const. This is the hyperbolic quasilinear system of equations. The properties of such systems are studied in detail (see, e.g., Refs. 29, 31). It is well-known that for a multitude of initial disturbances the corresponding smooth solutions to these equations exist only during finite time intervals. At the end of such an interval a gradient catastrophe occurs, i.e., infinite gradients develop. Then the plasma flow overturns resulting in the formation of a multiflow region. We illustrate this phenomenon by an example of a so-called simple waves.

Using a standard technique, ${ }^{29}$ we find that the magnetic field in a simple wave obeys the equation,

$$
\frac{\partial B_{y}}{\partial t}+V_{f}\left(B_{y}\right) \frac{\partial B_{y}}{\partial x}=0,
$$

which has a solution,

$$
x-V_{f}\left(B_{y}\right) t=F\left(B_{y}\right),
$$

where $F\left(B_{y}\right)$ is an arbitrary function of the magnetic field. The plasma density and velocity can be easily found using the Riemann invariants, which are constant in a region occupied by a simple wave. Since the thermal effects do not change the qualitative features of the phenomenon, they can be neglected for simplicity. In this case the Riemann invariants are

$$
\begin{aligned}
& R_{1}=\frac{\left(B_{x}^{2}+B_{y}^{2}\right)^{1 / 2}}{\rho}, \\
& R_{2}=V_{x} \pm\left(\frac{R_{1}}{\pi}\right)^{1 / 2}\left(B_{x}^{2}+B_{y}^{2}\right)^{1 / 4}, \\
& R_{3}=V_{y} \mp \frac{B_{x}}{2}\left(\frac{R_{1}}{\pi}\right)^{1 / 2} \int \frac{d B_{y}}{\left(B_{x}^{2}+B_{y}^{2}\right)^{3 / 4}} .
\end{aligned}
$$

The phase velocity of the wave is given by

$$
V_{f}=R_{2} \mp \frac{3}{2}\left(\frac{R_{1}}{\pi}\right)^{1 / 2}\left(B_{x}^{2}+B_{y}^{2}\right)^{1 / 4} .
$$

Choose the lower signs in Eqs. (5)-(7) to consider a wave, which propagates with respect to the plasma in the positive direction of the $x$-axis. From Eq. (3) it follows that an infinite gradient of the magnetic field (as well as the gradients of the other parameters describing the plasma flow) develops for the first time at

$$
t=\min \frac{F^{\prime}\left(B_{y}\right)}{V^{\prime}\left(B_{y}\right)} .
$$

This gradient catastrophe could happen if the dispersion would not counteract to prevent it. These effects cannot be described by the magnetohydrodynamic equations. When characteristic lengths become shorter than $c / \omega_{p i}$, one should change the model equations to take into account the dispersion due to two-fluid nature of the plasma.

\section{Dispersion effects and whistler-type precursor wave trains}

If the characteristic length of the flow becomes comparable with the dispersion length, the system goes to the region II on the dispersion curve (see Fig. 2), where its evolution is governed by a more complicated system of the twofluid magnetohydrodynamics.

If the initial disturbance has a small amplitude, its steepening will stop when the characteristic length of the region with the largest gradients attains the dispersion length 
$c|\cos \theta| / \omega_{p i}$ (as it was stated above, here and in the following we assume that the dissipation is weak, i.e., the dissipation results in the large scale variations of the plasma parameters, but it does not influence considerably the fine structure of the front). In this case the equations describing the evolution of the shock wave structure can be simplified by neglecting the electron inertia (see, e.g., Refs. 1, 32). Thereby we obtain ${ }^{32}$

$$
\begin{aligned}
& \frac{d \mathbf{V}}{d t}=\frac{1}{4 \pi \rho}[\operatorname{rot} \mathbf{B} \times \mathbf{B}], \\
& \operatorname{rot} \mathbf{B}=\frac{4 \pi n e}{c}\left(\mathbf{V}-\mathbf{V}_{e}\right), \\
& \frac{\partial \mathbf{B}}{\partial t}=\operatorname{rot}[\mathbf{V} \times \mathbf{B}]-\frac{m_{i} c}{e} \operatorname{rot} \frac{d \mathbf{V}}{d t}+\frac{m_{e} c}{e} \operatorname{rot} \frac{d \mathbf{V}_{e}}{d t},
\end{aligned}
$$

where $\mathbf{V}=\left(m_{e} \mathbf{V}_{e}+m_{i} \mathbf{V}_{i}\right) /\left(m_{e}+m_{i}\right)$ is the bulk plasma velocity, $\mathbf{V}_{e, i}$ are the fluid velocities of the electron and ion components.

Then we can introduce two small parameters,

$$
\varepsilon_{1}=\frac{\beta}{v_{A} l^{2}} \ll 1, \quad \varepsilon_{2}=\frac{B_{y}-B_{0} \sin \theta}{B_{0}} \ll 1,
$$

where $\beta=v_{A} c^{2} \cos ^{2} \theta / 2 \omega_{p i}^{2}, l$ is a characteristic length of the disturbance, $B_{0}$ is the unperturbed magnetic field, $\theta$ is the angle between the unperturbed magnetic field and the direction of the wave propagation.

Retaining the terms up to the second order with respect to these parameters, and considering only the waves propagating in the positive direction, after some algebra we obtain $^{32}$

$$
\frac{\partial b}{\partial t}+\left(v_{A}+\frac{3 v_{A} \sin \theta}{2 B_{0}} b\right) \frac{\partial b}{\partial x}-\beta \frac{\partial^{3} b}{\partial x^{3}}=0,
$$

where $b=B_{y}-B_{0} \sin \theta$. This equation is equivalent to Korteweg-de Vries equation, describing nonlinear waves with a positive dispersion. It is well-known that all solutions to Korteweg-de Vries equation have no sharp crests and do not break. This means that the dispersion of short waves is strong enough to prevent the growth of gradients due to nonlinear effects. However, this equation is valid only for small and smooth disturbances.

To analyze the evolution of large-amplitude disturbances, we should at least begin with the equations of twofluid magnetohydrodynamics, where the effects of electron inertia are also taken into account. It is generally believed that under some conditions these equations have no smooth solutions. In particular, it is well-known that stationary solutions can have sharp crests (see, e.g., Refs. 1, 2). In addition, we should expect that these equations describe also the wave breaking of the hyperbolic kind with the development of the vertical slope (a gradient catastrophe) and a multivalued profile. Up to now the rigorous proof of the corresponding mathematical theorem has not been obtained yet. However, we can suggest several arguments confirming this statement.
From the set of equations of the two-fluid magnetohydrodynamics, after a cumbersome but straightforward algebra of the reductive perturbation method ${ }^{33}$ we obtain a model equation,

$$
\frac{\partial u}{\partial t}+u \frac{\partial u}{\partial x}+\int_{-\infty}^{+\infty} K(x-\xi) \frac{\partial u(t, \xi)}{\partial \xi} d \xi=0 .
$$

This equation was proposed by Whitham ${ }^{34}$ as the simplest equation which combines two important factors, the typical hydrodynamical nonlinearity and dispersion of the arbitrary type. Indeed, the phase speed of the linear waves is given by the Fourier transform of the kernel of the integral operator,

$$
v_{\mathrm{ph}}(k)=\int_{-\infty}^{+\infty} K(x) \exp (-i k x) d x,
$$

and vice versa,

$$
K(x)=\frac{1}{2 \pi} \int_{-\infty}^{+\infty} v_{\mathrm{ph}}(k) \exp (i k x) d k .
$$

Later $^{35}$ it was realized that the energy dissipation and pumping can also be described by Eq. (9). Because the main goal of the paper is to study the role of the dispersion in the formation and breaking of the shock front, we neglect the dissipation in order to simplify the problem considered.

Whitham $^{34}$ formulated the conjecture that Eq. (9) with the kernel $K_{g}(x)$ describing the dispersion of water waves,

$$
v_{\mathrm{ph}}(k)=\left[\frac{g}{k} \tanh \left(k h_{0}\right)\right]^{1 / 2},
$$

where $g$ is the gravitational acceleration and $h_{0}$ is the depth of the water, has stationary solutions with sharp crests as well as breaking nonstationary solutions. He proved that peaking of stationary solutions takes place if $K(x)$ behaves like $|x|^{-\alpha}$ as $x \rightarrow 0$, where $\alpha>0$. Since in the vicinity of the origin the normalized kernel corresponding to $g=h_{0}=1$ has the asymptotics $K_{g}(x) \sim(2 \pi x)^{-1 / 2}$, the first part of the conjecture is thereby proved. The rigorous proof of the second part requires quite ingenious arguments and was given by Naumkin and Shishmarev. ${ }^{35,36}$ Moreover, they proved a more general theorem that a solution of the Whitham equation does break if the slope of the profile of an initial disturbance is sufficiently large and negative at some point and at the origin of coordinates the kernel of the integral operator has a singularity, which is weaker than $|x|^{-\alpha}$, where $1 / 2 \leqslant \alpha<3 / 5$ (the exact statement of the theorem can be found in Ref. 35 and is also given in Appendix A).

It seems to be quite natural that the order of singularity of the kernel is of particular importance. Indeed, breaking as well as peaking are small scale phenomena corresponding to large wave numbers. On the other hand, it is the short-wave part of the dispersion relation that determines the behavior of the kernel in the vicinity of the coordinate origin. The Korteweg-de Vries equation (8) takes $v_{\mathrm{ph}}=v_{A}+\beta k^{2}$ and

$$
K(x)=v_{A} \delta(x)-\beta \delta^{\prime \prime}(x)
$$

[here $\delta(x)$ is the Dirac delta-function] and is known to have neither peaking nor breaking solutions. In other words, the 
dispersion of short waves is sufficiently strong to prevent developing infinite gradients due to nonlinearity. On the contrary, if the kernel has no singularity at the origin and is a monotone decreasing function of $|x|$, Seliger ${ }^{37}$ proved that in this case the Whitham equation does have breaking solutions (see also Ref. 38). Since nonsingular integrable kernels satisfy the conditions of the above-mentioned theorem proved by Naumkin and Shishmarev, this theorem should be considered as a substantial generalization of the Seliger result.

Taking into account these considerations, in the following we use a simplified dispersion relation,

$$
v_{\mathrm{ph}}=\frac{|\cos \theta|}{\mu^{1 / 2}} \frac{|\kappa|}{1+\kappa^{2}},
$$

which is valid for $k c / \omega_{p i} \gg 1$. Here $v_{\mathrm{ph}}=\omega / k v_{A}$ is the dimensionless phase velocity and $\kappa=k c / \omega_{p e}$ is the dimensionless wave number. Substituting Eq. (11) into Eq. (10), after some straightforward calculations we obtain

$$
K(x)=-\frac{1}{2 \pi} \frac{|\cos \theta|}{\mu^{1 / 2}}[\exp (-x) \operatorname{Ei}(x)+\exp (x) \operatorname{Ei}(-x)],
$$

where $\operatorname{Ei}(x)$ is the exponential integral. Using the asymptotical expansion ${ }^{39}$

$$
\operatorname{Ei}(x) \sim C+\ln |x|+x+\cdots
$$

as $x \rightarrow 0$, we can easily obtain

$$
K(x) \sim-\frac{1}{\pi} \frac{|\cos \theta|}{\mu^{1 / 2}}[C+\ln |x|+o(x)],
$$

where $\mathrm{C}$ is the Euler constant. Since $\ln |x| /|x|^{-\alpha} \rightarrow 0$ as $x \rightarrow 0$ for all $\alpha>0$, the condition 1 of the theorem is satisfied (see Appendix A). It is easily seen that the condition 2 is also satisfied. Thus, nonlinear waves, which are described by the model Eq. (9) with the dispersion typical for whistler waves, do break like hyperbolic waves provided the initial disturbance is sufficiently steep (see condition 3 of the theorem in Appendix A).

Although the Whitham equation with the whistler dispersion relation is model rather than exact, it can qualitatively describe the gradient catastrophes within the front of the supercritical nonstationary shock waves. It is worth noting that the description of this system is quite similar to that of shallow water waves, for which similar theoretical conclusions were approved by direct experimental results.

Let us now proceed to more moderate disturbances that are not steep enough to satisfy the condition 3 of the Naumkin-Shishmarev theorem. Suppose further that in the system there exists some kind of dissipation provided by, for example, an anomalous resistivity. To take the dissipation into account, we can modify the kernel of the Whitham equation (see Ref. 35 and Appendix A for details). In this case the disturbance can asymptotically approach some shock-like solution with a steady profile. Because for oblique propagation the dispersion of the fast magnetosonic wave is positive, weak shocks have a wave-train precursor damping out upstream. ${ }^{1,2}$ To determine the wave number of these oscilla- tions far upstream of the shock "ramp," we should equate the upstream plasma velocity to the phase velocity given by the dispersion relation and solve the equation obtained. There exists an additional condition for a precursor to exist, namely, the group velocity should exceed the corresponding phase velocity (see Appendix B). Using Eq. (11) it can be easily shown that this condition is satisfied for all wave vectors within the range $0 \leqslant \kappa \leqslant 1$. The whistler phase velocity is the highest for $\kappa=1$, the corresponding Mach number is called the whistler critical Mach number ${ }^{17}$ and is given by

$$
M_{w}=\frac{\left|\cos \theta_{B n}\right|}{2 \mu^{1 / 2}} .
$$

It is well-known that the whistler precursor wave train is an essential part of the oblique subcritical shocks. ${ }^{1,2}$ The very similar precursors were also evidenced for the supercritical shocks. ${ }^{20,21}$ We suggest a new proof of the criteria for existence of the precursor and wake wave trains (see Appendix B). From this proof it is evident that these criteria are quite general, in particular, they hold not only for subcritical shock waves but for supercritical shocks as well, because a small fraction of reflected ions does not change considerably the dispersion relation for the whistler waves (see Appendix B for more details).

The precursor wave train predecelerates the plasma flow upstream of the ramp of the shock and makes the contribution to the energy dissipation. Karpman et al. suggested that this dissipation can be related to the parametric instability of the whistler waves ${ }^{40}$ and/or wave-particle interaction. ${ }^{41}$ If the Mach number exceeds $M_{\mathrm{w}}$, this mechanism is switched off and the other components of the shock front structure (e.g., the ramp) should provide stronger plasma flow deceleration and dissipation. This leads to the growth of gradients and as a result, the ramp of the shock is replaced by a soliton-like nonlinear whistler wave train with a characteristic wave length of about several $c / \omega_{p e}$. These waves were observed within the Earth and Uranian bow shocks. ${ }^{10,21}$ Using twofluid MHD equations, we analyze the properties of these waves in the plasma with cold ions, finite $\beta_{e}$, and adiabatic equation of state for electrons (see Appendix C). It was shown that the amplitude of these waves increases with Mach number and can significantly exceed the value of the magnetic field ahead of the shock. However, when Mach number exceeds the critical value $M_{\mathrm{nw}}$, such waves do not exist anymore. For cold plasma the corresponding Mach number, which can be referred to as a nonlinear whistler critical Mach number, is given by

$$
M_{\mathrm{nw}}=\frac{\left|\cos \theta_{B n}\right|}{(2 \mu)^{1 / 2}} .
$$

As it was shown above, the critical Mach number $M_{\mathrm{nw}}$ corresponds also to the characteristic Mach number above which an initial disturbance resembling the quasistationary whistler wave train becomes unstable with respect to a gradient catastrophe, which takes place within a finite time interval. Thereby we come to the conclusion that $M_{\mathrm{nw}}$ can be 


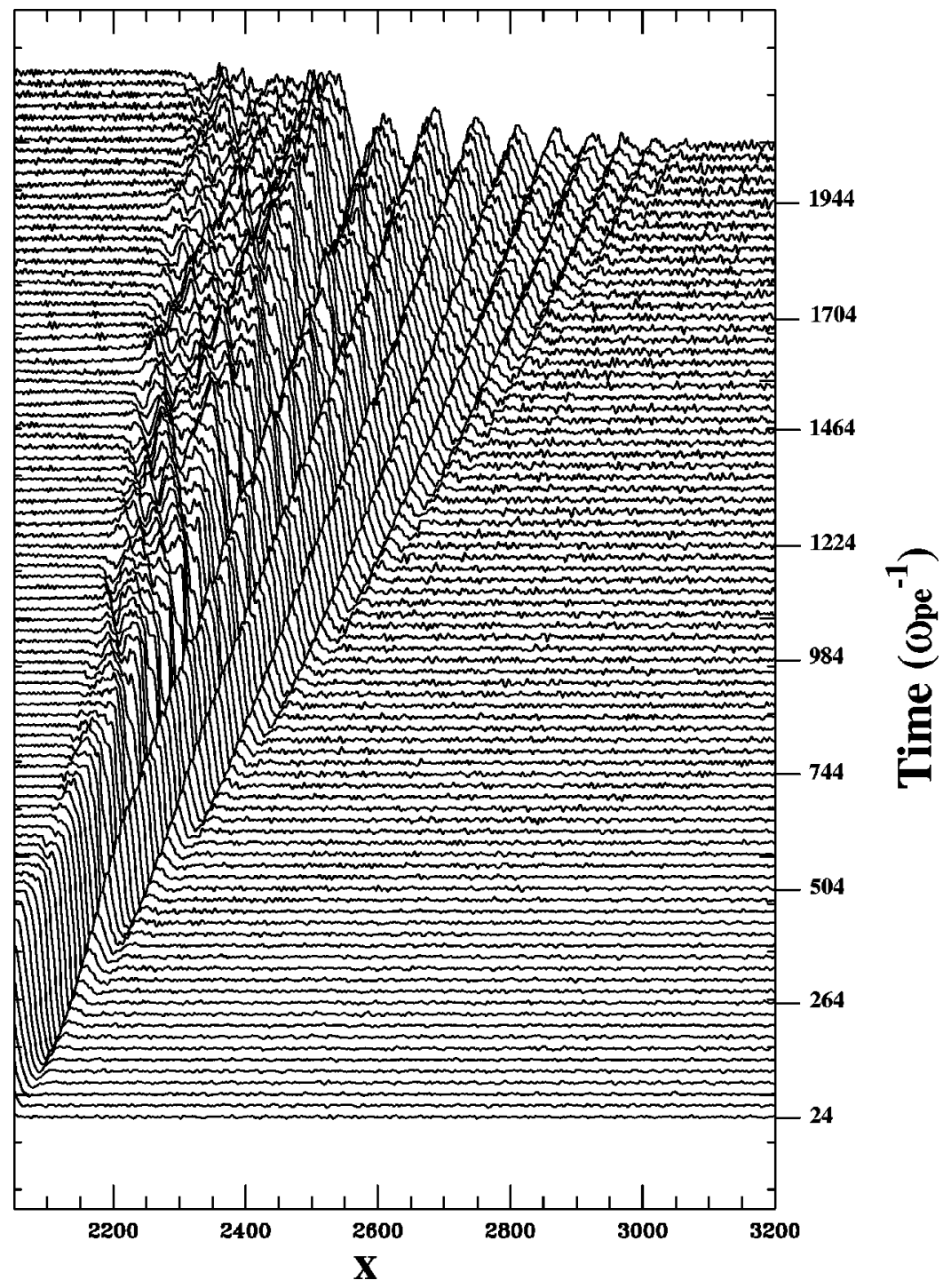

FIG. 3. The stackplot for the component $B_{z}$ of the magnetic field in the shock wave with $\theta_{B n}=57^{\circ}$ and $M_{A}$ $=2.7$.

taken as the estimate of the critical Mach number $M_{\mathrm{ns}}$ corresponding to the transition between stationary and nonstationary behavior of the shock front.

When $M_{\mathrm{nw}}$ is exceeded, the dispersion and dissipation due to reflected ions and anomalous resistivity are no longer sufficient to stop the wave steepening due to nonlinear effects and an additional mechanism of the energy dissipation is required. The additional dissipation can be provided by several effects accompanying the shock front nonstationarity. Indeed, a nonstationary shock should emit the whistler wave trains thereby evacuating the energy from the ramp. However, when the Mach number exceeds the value,

$$
M_{\mathrm{gr}}=\frac{\left|\cos \theta_{B n}\right|}{8}\left(\frac{27}{\mu}\right)^{1 / 2},
$$

corresponding to the maximum group velocity of the whistler waves, the evacuation of the energy far upstream becomes impossible. The short nonstationary whistler "precursor" can still exist in the foot, where the plasma flow is slightly decelerated, however, this wave train cannot propagate far upstream.
In the next section we present the results of full particle numerical simulations that give strong support to our statement that the transition from stationary to nonstationary behavior of the shock waves takes place when the Mach number becomes larger than $M_{\mathrm{nw}}$.

Lastly, we briefly dwell upon the role of reflected ions in the shock wave structure and dynamics. It is well-known that the behavior of the reflected ions determines the large scale structure of the quasistationary supercritical shock waves and supply the major part of the dissipation required (see, e.g., a review, Ref. 17). On the other hand, it is the ion dynamics that determine the time scale for quasiperiodic overturning of the high Mach number shocks. However, since the fraction of the reflected ions usually does not exceed 10\%-20\% for quasistationary shock waves, as a first approximation, we can neglect its contribution when deriving the criterion for transition between stationary and nonstationary shocks. Indeed, the corresponding critical Mach number is determined first of all by dispersion and nonlinearity of the fast magnetosonic waves and these properties are predominantly determined by the bulk particle populations. 


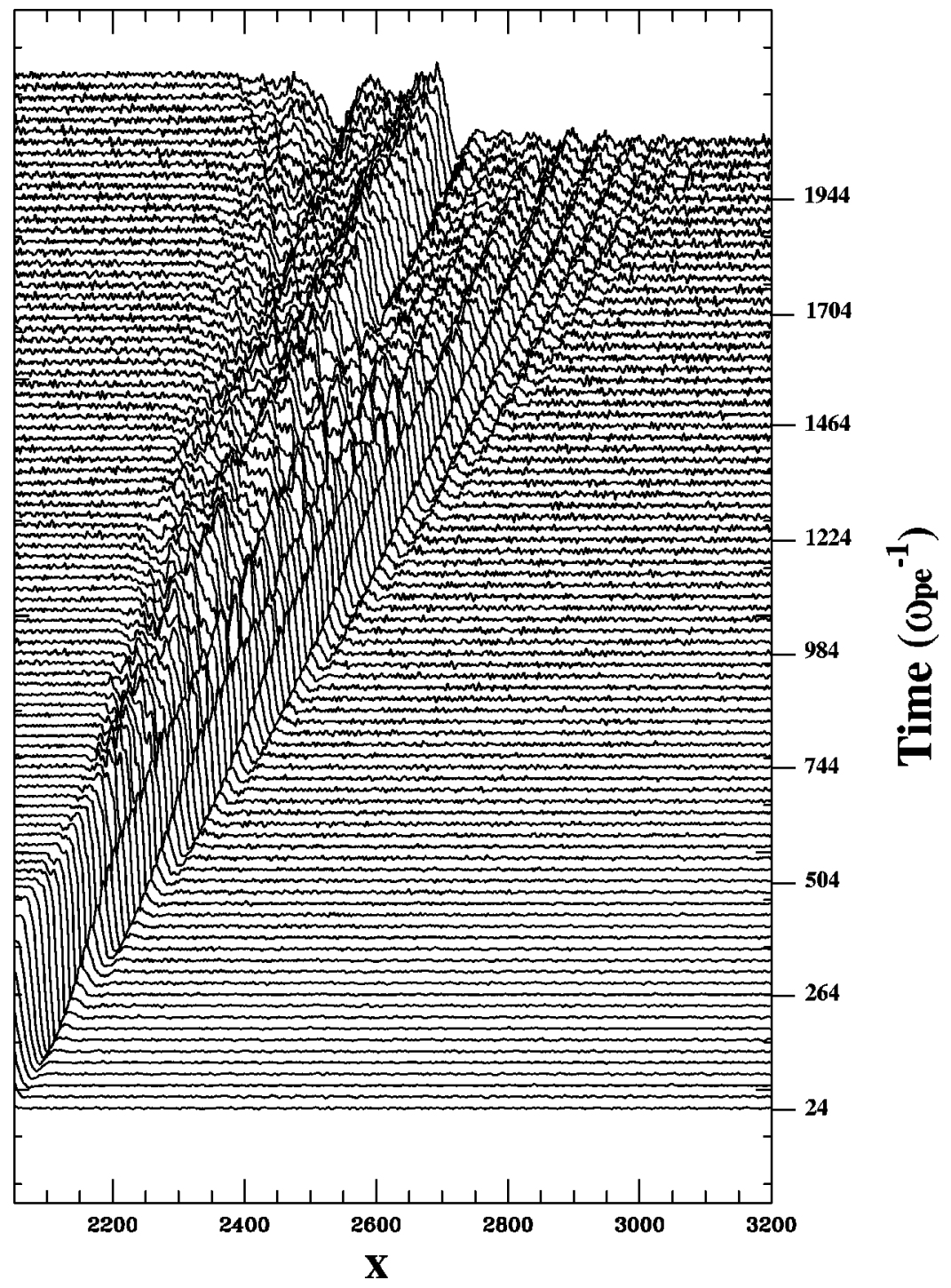

FIG. 4. The stackplot for the component $B_{z}$ of the magnetic field in the shock wave with $\theta_{B n}=57^{\circ}$ and $M_{A}$ $=3.3$.

\section{TRANSITION FROM STATIONARITY TO NONSTATIONARITY OF THE SHOCKS: NUMERICAL SIMULATIONS}

Present simulations have been performed by use of 1D fully electromagnetic relativistic particle code, where both electrons and ions are treated as particles. Standard finitesize particle techniques are used. ${ }^{42,43}$ All three velocity components for all particles are taken into account but the problem considered is one-dimensional, all the variables depending on $x$. The field components are separated into two groups, namely, the transverse components, $E_{y, z}$ and $B_{y, z}$, and longitudinal components, $E_{x}$ and $B_{x}$. To find the transverse components, the full set of Maxwell's equations is solved. Longitudinal component of the magnetic field $B_{x}$ is constant and the corresponding component of the electric field obeys Poisson's equation.

Initial and boundary conditions are similar to those already described previously. ${ }^{22,23}$ In short, the simulation box is separated into two adjacent parts, there is a vacuum in one of them and plasma in the another one. The latter box is bounded by reflecting walls preventing the plasma from penetrating into the former box. To drive a shock, a magnetic piston is generated by applying an external current pulse in the vacuum near the boundary between the boxes.

The spatial grid is uniform. All the quantities in the code are normalized. In particular, the length is measured in the spatial grid increments $a$, that is, the width of the cell. In the figures in the following, shown are the normalized differences between the $z$ component of the magnetic field and its upstream value. The normalization was performed by multiplying the differences by the factor $e / m_{e} \omega_{p e}^{2} a$, where $e$ is the proton charge. It is worth noting that for brevity we shall speak in the following about the magnetic field rather than the normalized difference. The ion momentum is normalized by dividing it by $m_{e} \omega_{p e} a$. More details related to the code can be found elsewhere. ${ }^{22}$

The present simulations are performed under the following conditions. Size of the simulation box containing the plasma is $\tilde{L}=8192$. Initially, there are $\tilde{n}_{e, i}=10$ particles in each cell. The ratios of the electron and ion temperatures and masses are $T_{e} / T_{i}=1.58$ and $\mu=m_{e} / m_{i}=0.005$, respectively. The ratio of thermal and magnetic pressures is $\beta$ $=0.028$.

In the simulations performed by Leroy ${ }^{13}$ it was shown 


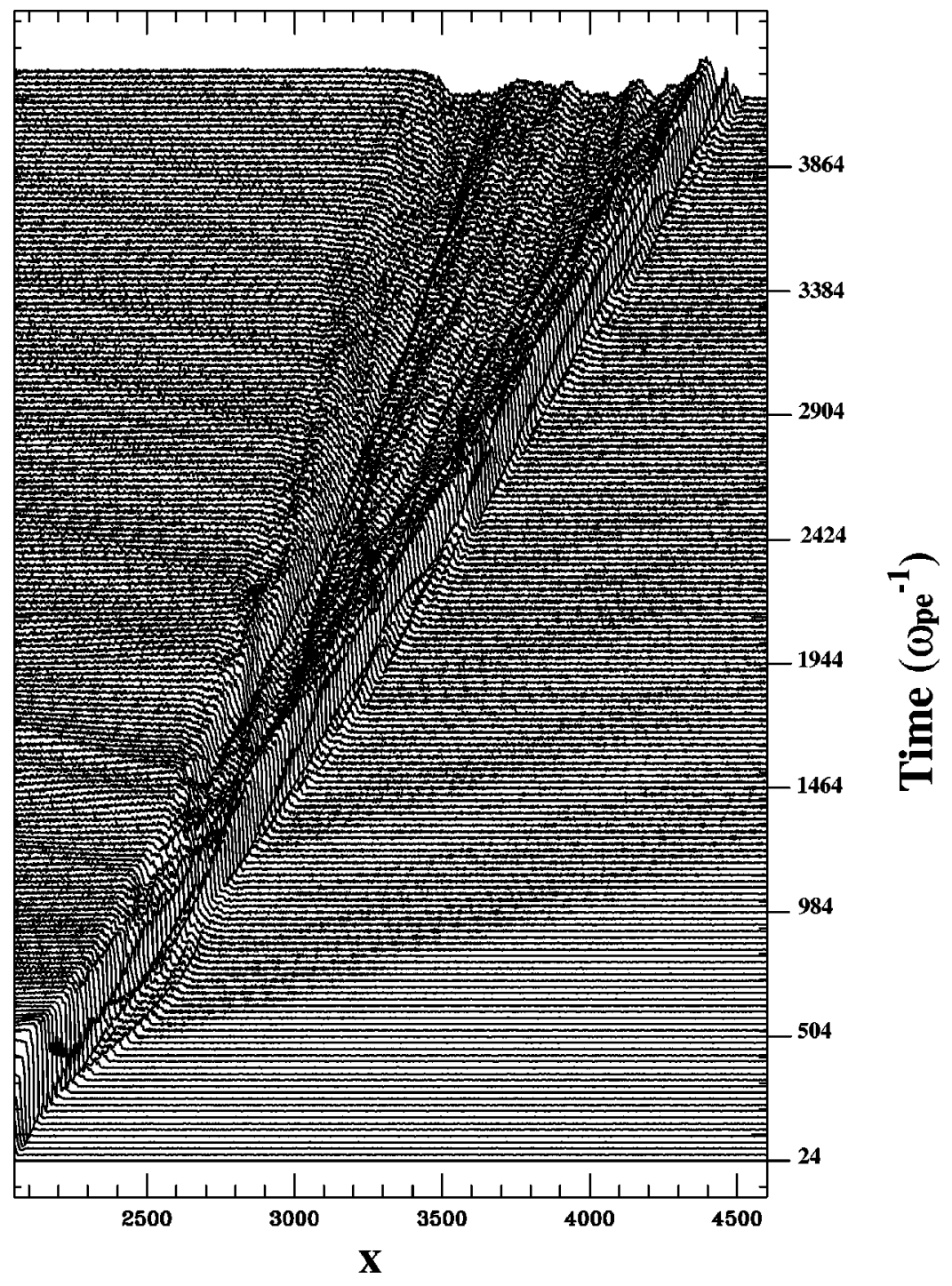

FIG. 5. The stackplot for the component $B_{z}$ of the magnetic field in the shock wave with $\theta_{B n}=57^{\circ}$ and $M_{A}$ $=5.5$.

that a self-sustained shock is formed after a transitory period lasting about several ion gyroperiods calculated with the use of downstream magnetic field. In the present simulations the total run time covers about 4 ion gyroperiods calculated with the use of upstream magnetic field.

As it was noted above, there exists a close relationship between the properties of linear waves and the structure of the shock waves in plasmas. ${ }^{1,2}$ In particular, the dispersion relation influences the shape of the subcritical shock waves. In numerical modeling, a special care should be taken to avoid the distortion of the dispersion relation due to the finite-difference approximation of the equations solved. ${ }^{11}$ Because the role of whistler waves in the formation of shock wave structure is of special interest, the discretization should not distort the dispersion relation for fast magnetosonic waves with the wave numbers within the range $0 \leqslant k c / \omega_{p e}$ $\leqslant 3$. Note that the phase velocity of the waves is maximum at $k \simeq \omega_{p e} / c$. To estimate the influence of the finitedifference approximation on a dispersion relation, one can multiply the phase velocity by a factor $\sin (k a) / k a$, where $a$ is the grid increment. ${ }^{11,44}$ In the present paper we choose $a$ $=c / 3 \omega_{p e}$. The direct calculations show that these effects in the range mentioned above can be considered as nonsignificant in this case.

Two series of runs were performed, where the angle $\theta_{B n}$ between the shock normal and the magnetic field upstream of the shock is equal to $57^{\circ}$ and $80^{\circ}$, respectively, and Mach number varies in the wide range from about 1.6 to 8.6 in the both series. Using the simulation results, we describe the evolution of the shock wave structure as the Mach number increases.

We begin the analysis from the $\theta_{B n}=57^{\circ}$ shock waves. In this case the first critical Mach number is $M_{\mathrm{cr}} \approx 2.54$, the linear and nonlinear whistler critical Mach numbers are $M_{\mathrm{w}}$ $\approx 3.85, M_{\mathrm{nw}} \approx 5.45$, respectively.

In the stackplot shown in Fig. 3 we observe the evolution of the magnetic field profile of the supercritical shock, $M_{A} \simeq 2.7$. Although the shock is supercritical, the critical Mach number is only slightly exceeded and the fraction of reflected ions, which are responsible for a typical structure of supercritical shocks, is quite small in the case considered. The quasisteady "ramp" is formed at the very beginning of the computational run. However, the wave train precursor expands upstream up to at least $t>2 \pi / \omega_{B i}$ with a higher 
speed than that of the shock ramp. This result is quite natural because the critical whistler Mach number is not exceeded and the stationary shock wave should have a wave train precursor standing upstream of the ramp.

The next example is a quite typical supercritical shock of Alfvén Mach number $M_{A} \simeq 3.3$. In the stackplot for $B_{z}$ component of the magnetic field we can clearly see the formation of the ramp, overshoot, and undershoot (see Fig. 4). The other characteristic feature of supercritical shocks, the foot, is also observed when analyzing the magnetic field profiles. In the foot region of the shock there is a wave train, which modulates the mean ion velocity and predecelerates the incoming flow. It is worth noting that in the case considered both linear and nonlinear whistler critical Mach numbers are not exceeded and the shock front structure is almost stationary.

For higher Mach numbers, $M_{A} \simeq 5.5$ and 8.6, when both the first critical Mach number and the nonlinear whistler Mach number are exceeded, the shock waves are nonstationary. For the shock wave with $M_{A} \simeq 5.5$, stackplot is shown in Fig. 5. Similar nonstationarity of the shock front is also evidenced for higher Mach numbers. Figure 6 illustrates the cycle of the reformation of the magnetic field structure with the emission of the whistler wave train towards the upstream flow. During the cycle, a new ramp is formed at the forward edge of the precursor wave. Here a small-amplitude perturbation grows up and becomes larger than the old shock front.

Now we proceed to shock waves with $\theta_{B n}=80^{\circ}$. In this case the first critical Mach number is $M_{\mathrm{cr}} \approx 2.74$, the whistler linear and nonlinear critical Mach numbers are $M_{\mathrm{w}} \approx 1.23$ and $M_{\mathrm{nw}} \approx 1.74$.

The stackplot in Fig. 7 shows the evolution of the magnetic field profile for shock wave with a relatively low Mach number, $M_{A} \simeq 1.6$. Since $M_{A}<M_{\mathrm{cr}}$, this shock is subcritical and the fraction of reflected ions is negligible. We observe that the "ramp" can be considered as almost stationary for $t>0.2 \cdot 2 \pi / \omega_{B i}$. Because the whistler critical Mach number is exceeded, there is no stationary wave train upstream of the ramp. However, the maximum group velocity of whistler waves is greater than the velocity of shock wave propagation, thus, a nonstationary wave precursor may be observed upstream of the ramp, at least, at the first stages of the shock formation.

In the next example we consider the supercritical shock with $M_{A} \simeq 3.5$. In Fig. 8 presenting the evolution of the magnetic field profile, it is easily seen that the shock is nonstationary and a quasiperiodic reformation of the shock front is observed. The scenario of the reformation is essentially the same as for high Mach number shocks with $\theta_{B n}=57^{\circ}$. At the first stage shown, the shock has a clearly defined ramp and upstream of the ramp there is a leading "wave train" of a small amplitude (see also Fig. 9). A population of reflected ions is observed between the ramp and the peak of the leading wave train (see the ion phase space display in Fig. 9). In this case the whistler precursor consists of only one peak
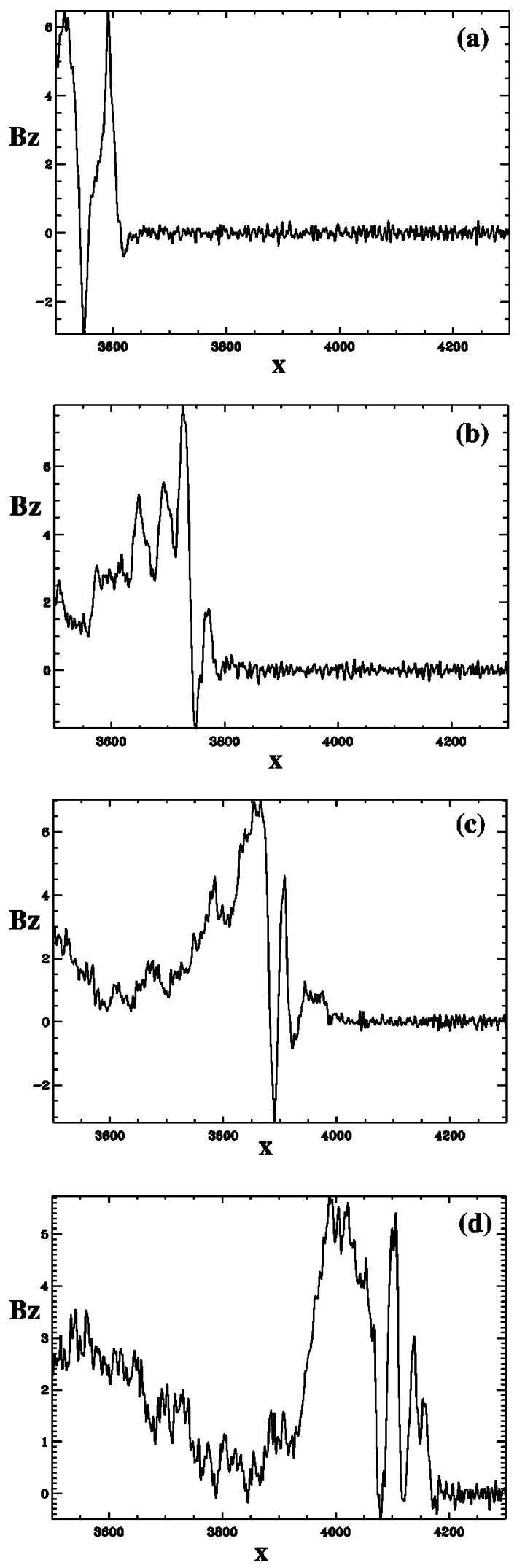

FIG. 6. The magnetic field profiles for a shock wave with $\theta_{B n}=57^{\circ}$ and $M_{A}=5.5$ at (a) $t=2496 \omega_{p e}^{-1}$, (b) $t=2808 \omega_{p e}^{-1}$, (c) $t=3096 \omega_{p e}^{-1}$, and (d) $t=3480 \omega_{p e}^{-1}$. 


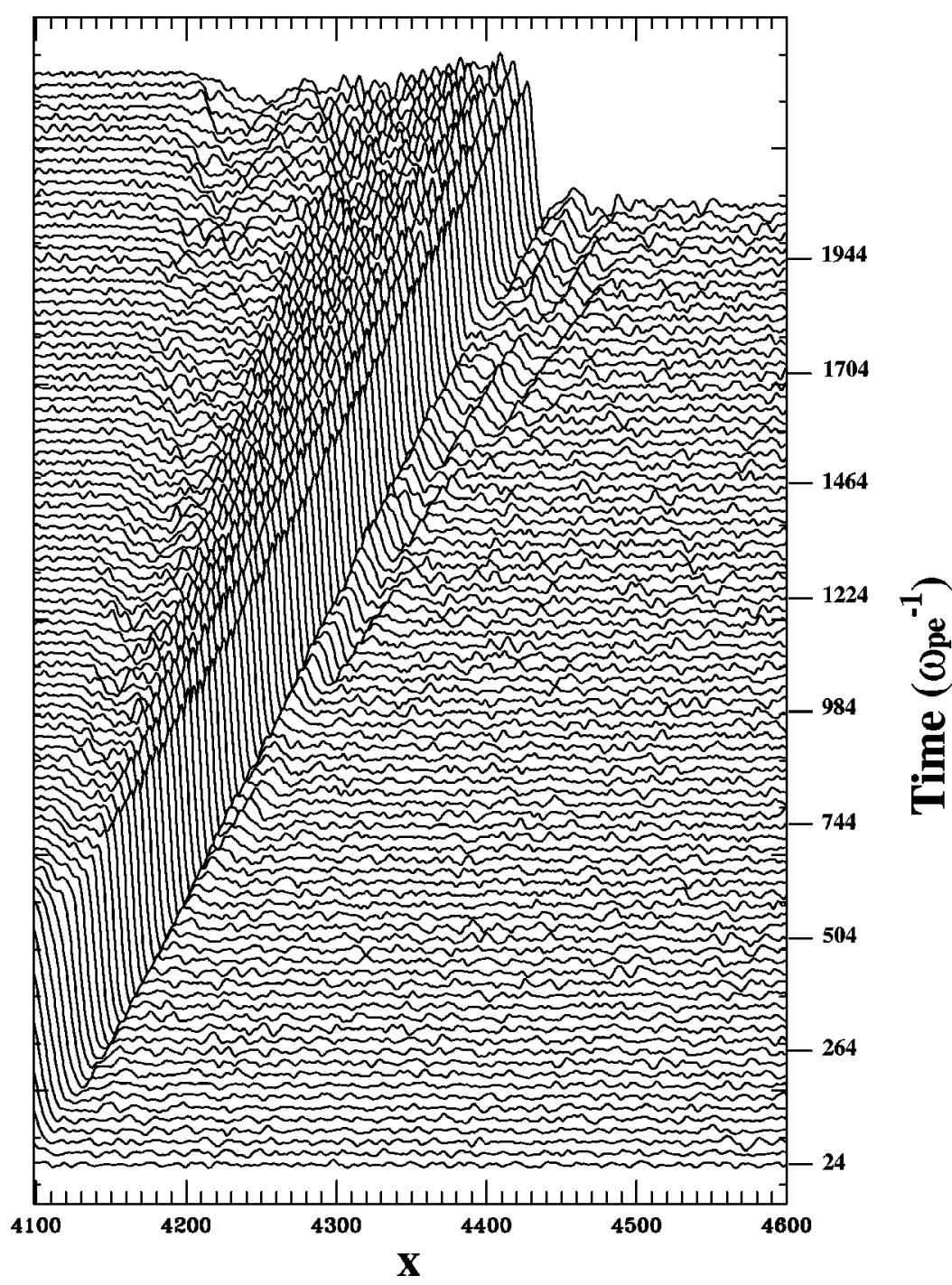

FIG. 7. The stackplot for the component $B_{z}$ of the magnetic field in the shock wave with $\theta_{B n}=80^{\circ}$ and $M_{A}$ $=1.6$.

because in the simulation the whistler phase velocity along the magnetic field has the same order of magnitude as the thermal speed of electrons, so that the damping of the whistlers is considerable. The amplitude of the leading wave train is gradually increasing. At some time a new population of reflected ions appears upstream of the precursor (see Fig. 10). When its amplitude becomes comparable with that of the ramp and, finally, exceeds it, thereby a new ramp and a new precursor are formed (see Figs. 11 and 12). The processes described are repeated quasiperiodically.

Finally, the results of the simulations for both chosen values of $\theta_{B n}$ confirm our statement that the transition from stationary to nonstationary behavior of the quasiperpendicular shock occurs when nonlinear whistler critical Mach number is exceeded.

\section{CONCLUSION}

In the present paper we study the problem of the nonstationarity of quasiperpendicular high Mach number shocks. Previously, Kennel et al. ${ }^{17}$ introduced a critical Mach number $M_{\mathrm{ns}}$ above which shock waves become nonstationary.
However, up to now the estimates of $M_{\mathrm{ns}}$ were not suggested. In the present paper, $M_{\mathrm{ns}}$ is determined for oblique quasiperpendicular fast magnetosonic shock waves.

Theoretical analysis and experiments show that the whistler waves are an intrinsic feature of the oblique collisionless shock waves. For supercritical shock waves, the ramp region, where an abrupt increase of the magnetic field occurs, can be treated as a nonlinear whistler wave of large amplitude. In addition, oblique shock waves can possess a linear whistler precursor. There exist two critical Mach numbers related to the whistler components of the shock wave, the first is known as a whistler critical Mach number introduced by Kennel et al., ${ }^{17} M_{\mathrm{w}}$, and the second can be referred to as a nonlinear whistler critical Mach number, $M_{\mathrm{nw}}$. It is worth noting that $M_{\mathrm{w}}<M_{\mathrm{nw}}$. When the whistler critical Much number is exceeded, a stationary linear wave train cannot stand ahead of the ramp. Above the nonlinear whistler critical Mach number, the stationary nonlinear wave train cannot exist anymore within the shock front. In this case the dispersion cannot prevent steepening of the shock front due to nonlinear effects and a gradient catastrophe occurs. As a result, the shock wave becomes nonstationary. Using model 


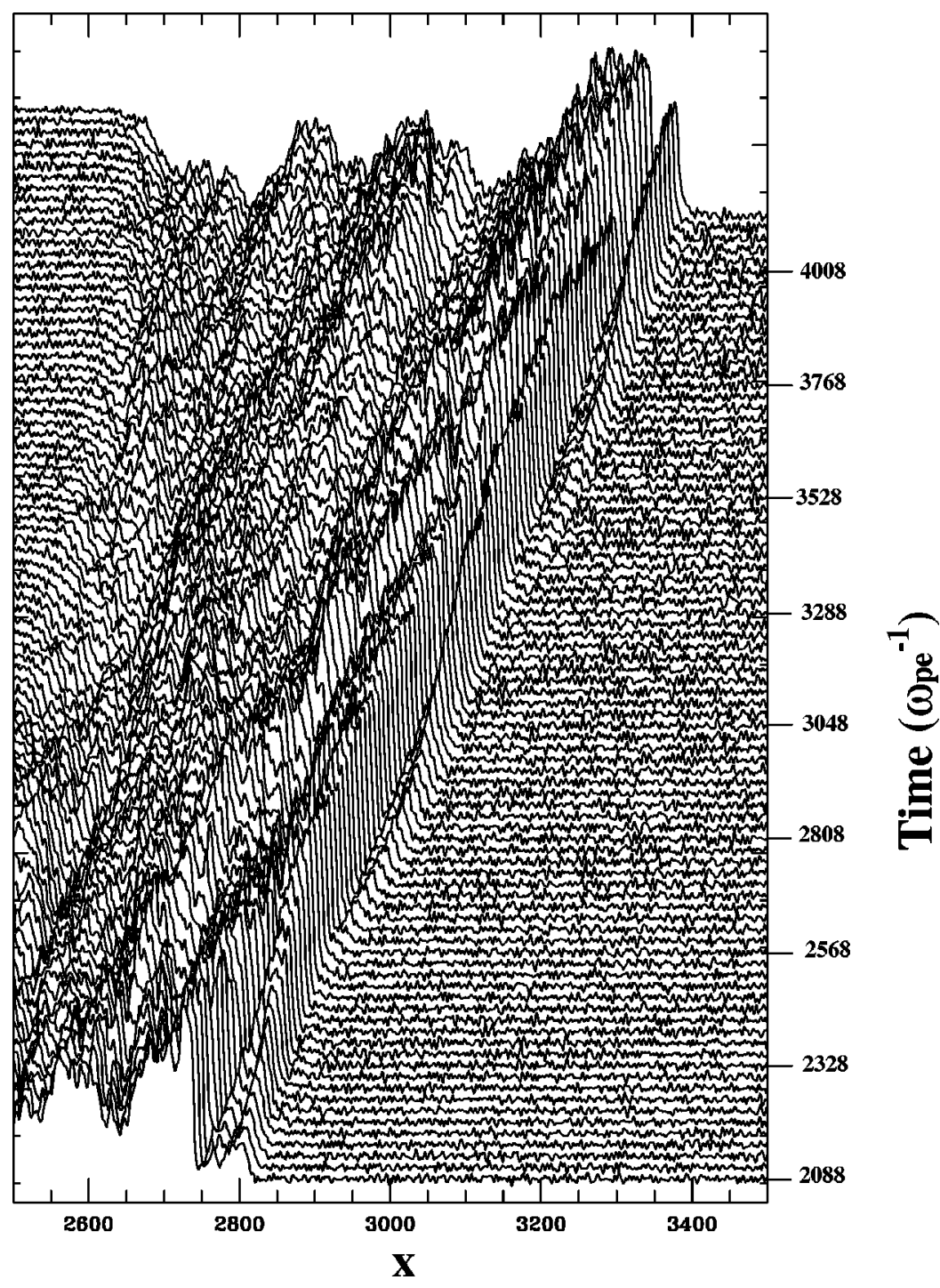

FIG. 8. The stackplot for the component $B_{z}$ of the magnetic field in the shock wave with $\theta_{B n}=80^{\circ}$ and $M_{A}$ $=3.5$.

equation description, we carried out the analysis of the dynamics of oblique shock waves and found that this equation does describe the wave breaking of the hyperbolic kind with the development of the vertical slope and a multivalued profile.

To obtain the $\beta$ - and $\theta_{B n}$-dependencies of $M_{\mathrm{nw}}$ corresponding to the transition between the stationary and nonstationary behavior of the shock wave, in the framework of the two-fluid magnetohydrodynamics we study the structure of nonlinear whistlers in plasmas with finite electron beta and adiabatic equation of state (see Appendix C).

In accordance with the results of the theoretical analysis, numerical simulations making use of the 1D full particle electromagnetic code demonstrated that the transition to the nonstationarity is always accompanied by the disappearance of the stationary whistler wave train within the shock front, i.e., the nonlinear whistler critical Mach number $M_{\mathrm{nw}}$ is closely related to $M_{\mathrm{ns}}$. The simulations show that front of the high Mach number shocks overturns quasiperiodically and the dynamics of the whistler wave trains within the front are of particular importance as was supposed previously. ${ }^{11,20}$ Recent experimental observations ${ }^{45}$ of the nonstationary whistler waves in the vicinity of the Earth bow shock confirm this model of the shock front nonstationarity.

In addition, we suggested a new proof of the criteria for small-amplitude linear precursor or wake wave trains to exist (see Appendix B). It was also shown that these criteria are quite universal and can be considered as necessary and sufficient conditions that turn out to be independent on both the nature of the waves and dissipative effects provided the medium far ahead and far behind the shock is stable and the dissipation is weak enough for weakly damping waves to exist.

\section{ACKNOWLEDGMENTS}

The authors would like to acknowledge the International Space Science Institute (Bern, Switzerland) for the financial support and the welcome of the working group "Multidimensional aspects of particle dynamics through the Earth's collisionless shock." V.V.L. is grateful to the administration of the Region Centre of France for financial support during his stay at LPCE (Orléans, France). He also acknowledges the warm hospitality of LPCE. 

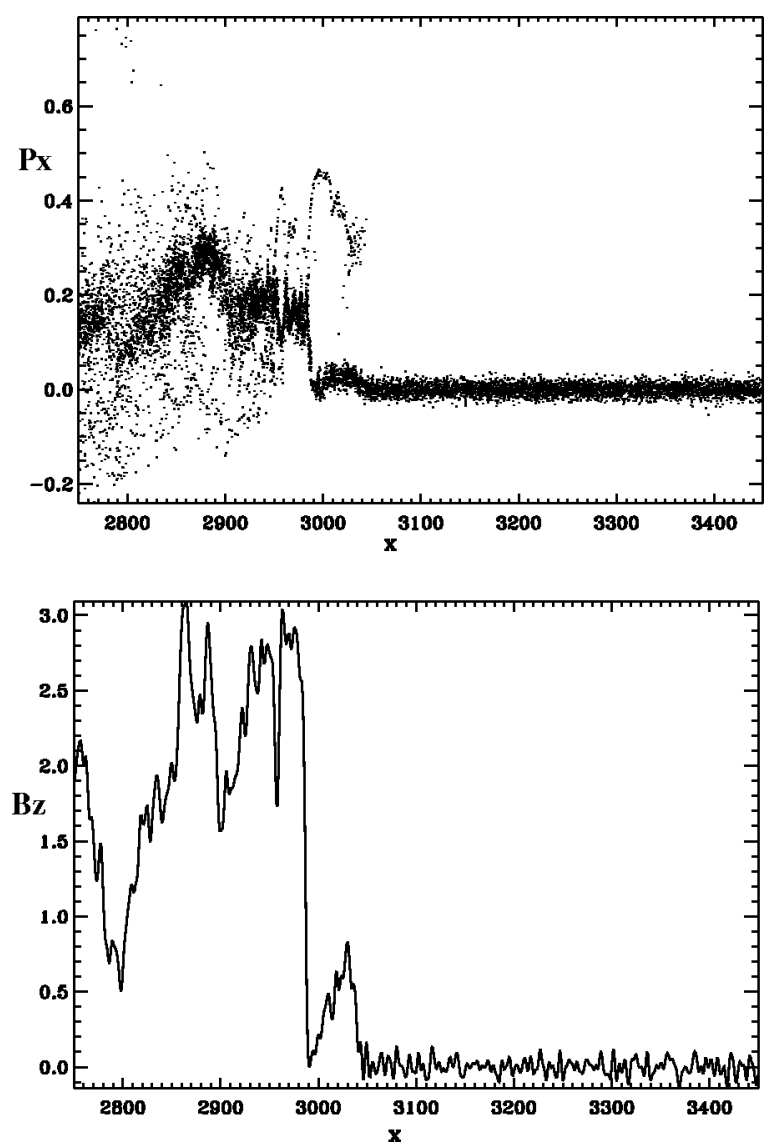

FIG. 9. The magnetic field profile (bottom panel) and $P_{x}-x$ phase space (top panel) for a shock wave with $\theta_{B n}=80^{\circ}$ and $M_{A}=3.5$ at $t=2860 \omega_{p e}^{-1}$.

All present numerical simulations have been performed on supercomputers of IDRIS center (Orsay, France).

\section{APPENDIX A: GRADIENT CATASTROPHE FOR THE WHITHAM EQUATION}

Consider the Whitham equation,

$$
\frac{\partial u}{\partial t}+u \frac{\partial u}{\partial x}+\mathcal{K} u=0,
$$

where $\mathcal{K}$ is a linear pseudodifferential operator. This operator can be written in two forms,

$$
\mathcal{K} u=\int_{-\infty}^{+\infty} K(x-\xi) \frac{\partial u(t, \xi)}{\partial \xi} d \xi
$$

or

$$
\mathcal{K} u=\frac{1}{2 \pi} \int_{-\infty}^{+\infty} \exp (i k x) \hat{K}(k) \hat{u}(t, k) d k,
$$

where

$$
\hat{u}(t, k)=\int_{-\infty}^{+\infty} \exp (-i k x) u(t, x) d x
$$

and $\hat{K}$ is called a symbol of the operator $\mathcal{K}$. Let $\hat{K}^{1}=\mathfrak{R}(\hat{K})$ and $\hat{K}^{2}=\Im(\hat{K})$. If only real solutions to the Whitham equa-
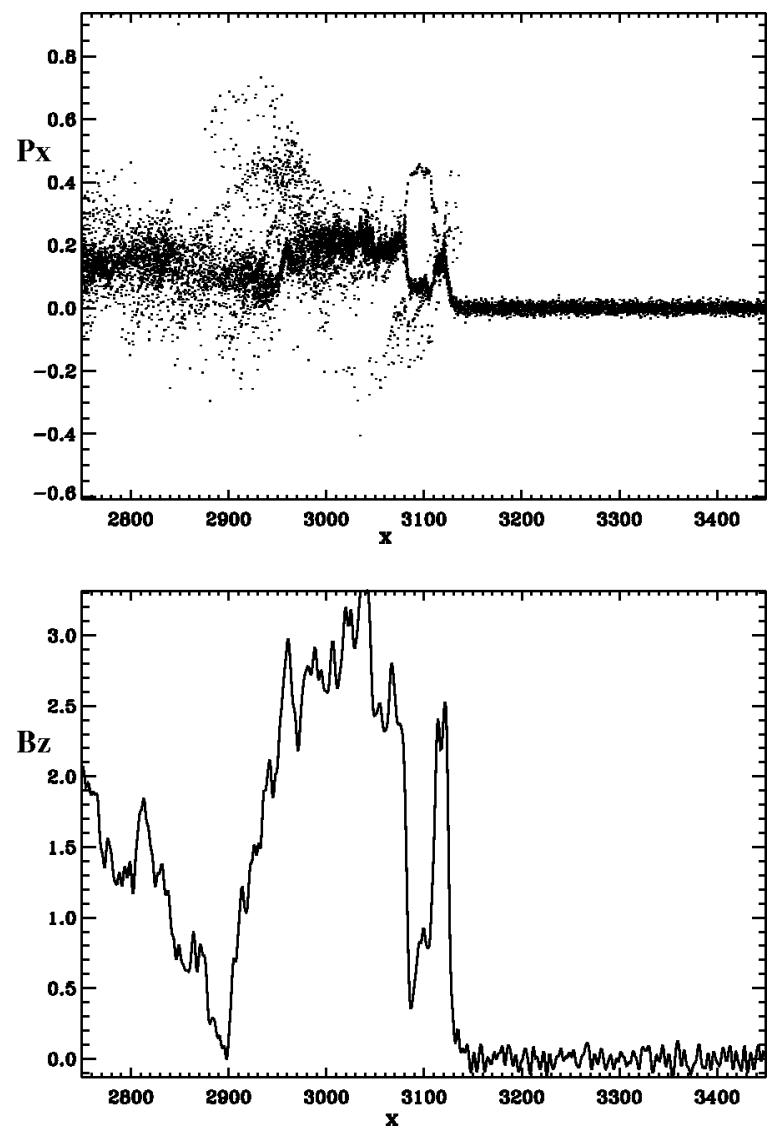

FIG. 10. The magnetic field profile (bottom panel) and $P_{x}-x$ phase space (top panel) for a shock wave with $\theta_{B n}=80^{\circ}$ and $M_{A}=3.5$ at $t=3240 \omega_{p e}^{-1}$.

tion are considered, $\hat{K}^{1}$ and $\hat{K}^{2}$ are called the dissipative and conservative parts of the symbol $\hat{K}$, respectively.

For the Whitham equation Naumkin et al. ${ }^{35}$ proved the following:

Theorem: Suppose that

(1) the kernel $K(x)$ satisfies the conditions

$$
\begin{aligned}
& K(x) \in C^{1}\left(R_{1} \backslash 0\right) \cap L_{1}\left(R_{1}\right), \quad|K(x)| \leqslant c|x|^{-\alpha}, \\
& \left|K^{\prime}(x)\right| \leqslant c|x|^{-1-\alpha}, \quad x \in[-a, a] \backslash 0, \\
& \int_{|x| \geqslant a}\left|K^{\prime}(x)\right| d x \leqslant c,
\end{aligned}
$$

where

$$
\alpha=3 / 5-\gamma, \quad \gamma \in(0,1 / 10], \quad c>0, \quad a \in(0,1] ;
$$

(2) the integral operator is dissipative, i.e., the symbol of the operator satisfies the inequality

$$
\hat{K}^{1}(p) \geqslant 0 \quad \text { for }|k| \geqslant h>0 ;
$$

(3) the initial perturbation $\bar{u}(x)$ belongs to the Sobolev space $H^{\infty}\left(R^{1}\right)$ and has a sufficiently large steepness $m_{0}$ $=\left|\min _{x \in R_{1}} \bar{u}^{\prime}(x)\right|$,

$$
m_{0}^{2}>\frac{7 c}{\gamma a}\left(u_{1}+\sqrt{J}\right)+\left(\frac{2 b}{\gamma}\right)^{2},
$$

where 

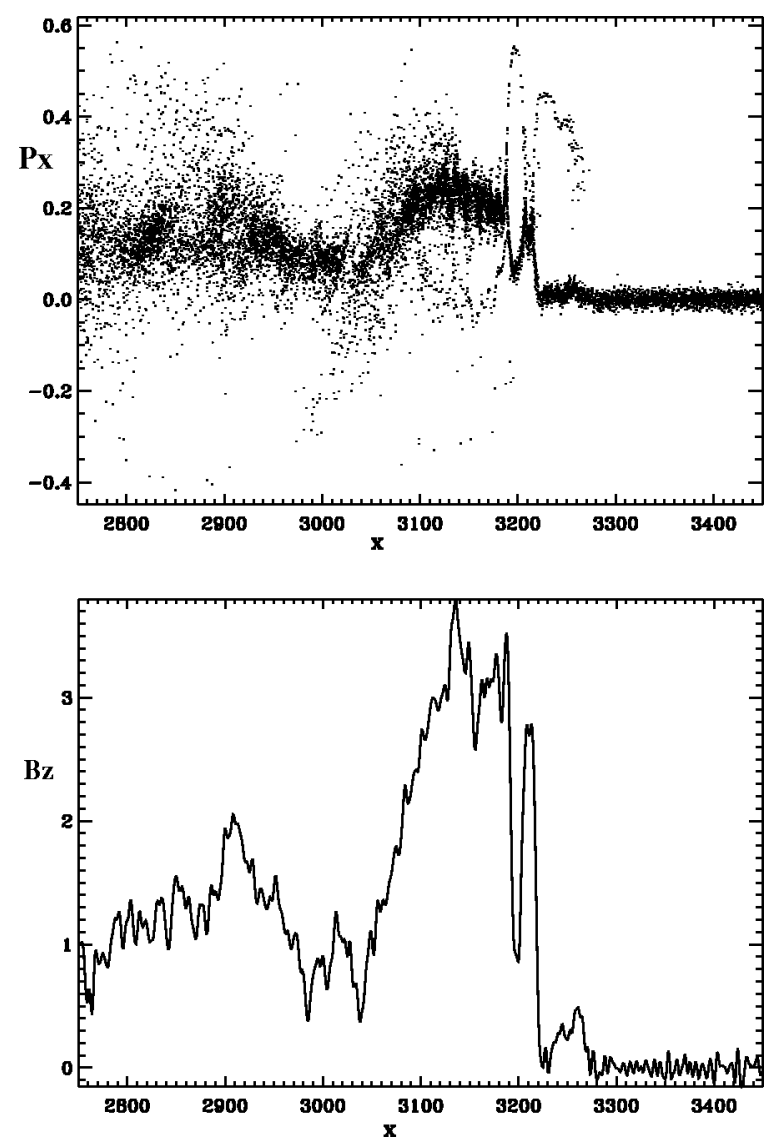

FIG. 11. The magnetic field profile (bottom panel) and $P_{x}-x$ phase space (top panel) for a shock wave with $\theta_{B n}=80^{\circ}$ and $M_{A}=3.5$ at $t=3624 \omega_{p e}^{-1}$.

$$
\begin{aligned}
& b=\sup _{|k| \leqslant h}\left(0,-\hat{K}^{1}(k)\right), \quad u_{1}=\max _{x \in R_{1}}\left|\bar{u}^{\prime}(x)\right|, \\
& J=\int_{-\infty}^{+\infty}\left(\frac{d^{3} \bar{u}(x)}{d x^{3}}\right)^{2} d x .
\end{aligned}
$$

Then there exists a solution $u(t, x)$ $\in C^{\infty}\left(\left[0, T_{0}\right) ; H^{\infty}\left(R_{1}\right)\right)$ breaking at the moment of time $T_{0}$. The following two-sided inequalities hold for $T_{0}$ :

$$
m_{0}^{-1}(1+\gamma)^{-1} \leqslant T_{0} \leqslant m_{0}^{-1}(1-\gamma)^{-2} .
$$

\section{APPENDIX B: PRECURSORS AND WAKES}

Suppose that the dissipation is negligible and the waves considered have a dispersion relation $\omega=\omega(k)$. Then the phase and group velocities are

$$
v_{\mathrm{ph}}=\frac{\omega}{k}, \quad v_{\mathrm{gr}}=\frac{\partial \omega}{\partial k},
$$

respectively. When considering a precursor (wake), suppose further that the velocity of the plasma far ahead (behind) the shock is $U_{1,2}$ in the frame where the shock is at rest. Then a steady precursor (wake) wave train can stand in the flow provided there exists a wave number $k_{0}$ such that

$$
\left.\frac{\omega}{k}\right|_{k=k_{0}}=U_{1,2},
$$
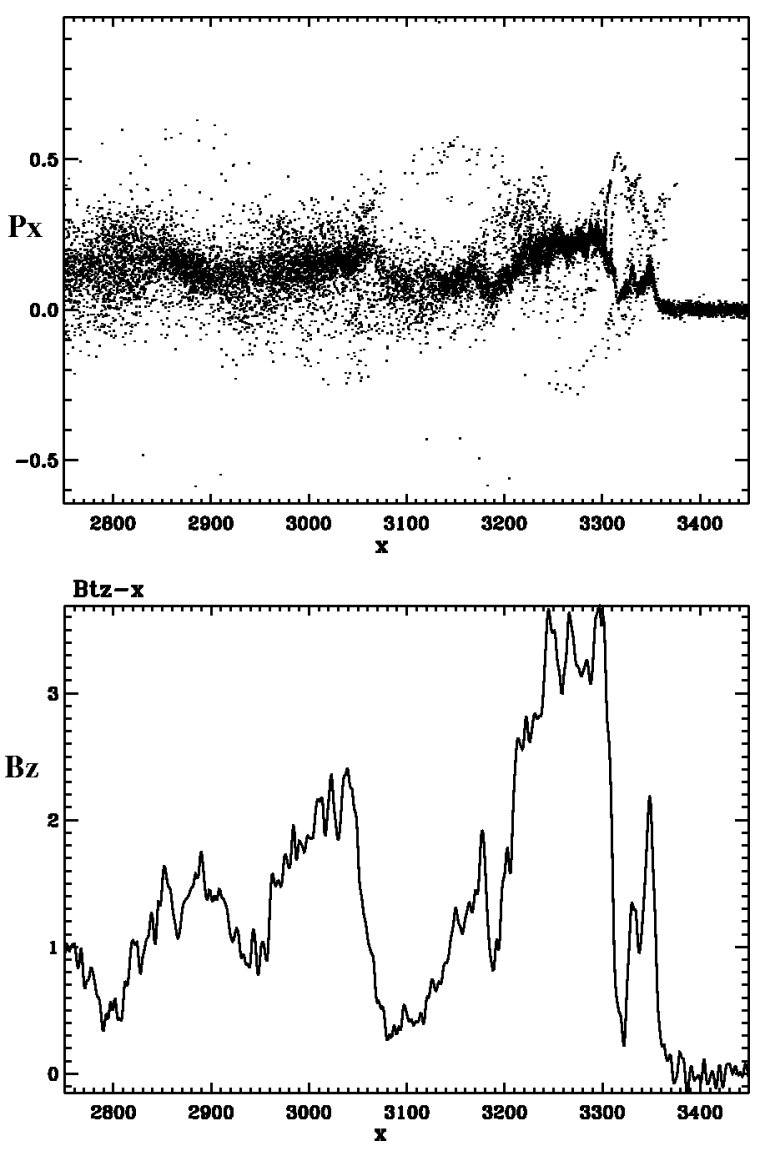

FIG. 12. The magnetic field profile (bottom panel) and $P_{x}-x$ phase space (top panel) for a shock wave with $\theta_{B n}=80^{\circ}$ and $M_{A}=3.5$ at $t=4008 \omega_{p e}^{-1}$.

and in addition

$$
\left.\frac{\partial \omega}{\partial k}\right|_{k=k_{0}}>\left.\frac{\omega}{k}\right|_{k=k_{0}}
$$

for a precursor and

$$
\left.\frac{\partial \omega}{\partial k}\right|_{k=k_{0}}<\left.\frac{\omega}{k_{0}}\right|_{k=k_{0}}
$$

for a wake. The condition (B1) is trivial - the phase velocity of the waves considered equals the velocity $U_{0}$ of the flow. The second criterion (B2) also becomes obvious if we consider a formation of a shock wave from a discontinuity satisfying the Rankine-Hugoniot conditions and to this end choose a reference frame where the shock is stationary. When emitting the waves both upstream and downstream, this step-like disturbance will be transformed into a steady shock with a precursor and/or wake wave trains. Since the wave number like the energy of the waves is transferred with the corresponding group velocity (see, e.g., Ref. 34), the waves can penetrate upstream and form a precursor if their group velocity exceeds the flow velocity ahead of the shock. To be convected downstream thereby forming a wake, the waves should have the group velocity which is less than that of the flow behind the shock.

It appears that criteria for a steady wave train precursor or wake of the shock wave to exist were developed for the 
first time by Tidman et al. ${ }^{46}$ To prove the criteria, they consider a model shock wave, which was represented by an infinite plane discontinuity, and a model dispersion relation in the form,

$$
D(\omega, k)=\prod_{i}\left[\omega-\omega_{i}(k)+i \varepsilon\right],
$$

where a small positive $\varepsilon$ describes a weak dissipative effects. They suppose that the electric current within the shock transition oscillates periodically, and using the linearized Vlasov-Maxwell equations, calculate the electromagnetic field generated by this current. The relationships obtained are rather cumbersome, but the above mentioned criteria follow from them immediately. Perez et al. ${ }^{47}$ carried these calculations further using the exact dispersion relation for a cold plasma.

As was shown above, the criteria (B1) and (B2) are easy to understand from the physical point of view and can be considered as necessary conditions for a precursor and/or wake to exist for shock waves in a plasma as well as in other media. However, the question remains as to the corresponding sufficient conditions. Indeed, using a stationary point analysis ahead and behind the shock waves in the dissipative magnetohydrodynamics, Coroniti ${ }^{48}$ showed that the different dissipative effects (resistivity, thermoconductivity, and viscosity) are not equivalent in the formation of the shock waves, e.g., the viscosity alone cannot form a fast MHD shock wave but the resistivity can. On the other hand, the criteria (B1) and (B2) does not contain any information about dissipation. One can suggest the two possibilities. First, these criteria are universal and sufficient for a precursor (wake) to exist upstream (downstream) the dispersive shock wave no matter what kind of waves is considered and what kind of dissipation is significant. Second, these criteria are only necessary and in each case Eq. (B2) should be replaced by a more strong condition, which is characteristic for a particular kind of the waves and/or dissipation. It can be easily shown that valid is the first statement.

To begin with, suppose that all parameters describing a wave depend upon time and coordinates like $f=f_{0}$ $\times \exp (-i \omega t+i k x)$ and the dispersion relation for these waves is

$$
D(\omega, k)=0 .
$$

If the dissipation is present but weak for the waves considered, Eq. (B3) can be written approximately as

$$
D_{0}(\omega, k)+D_{1}(\omega, k)=0 \text {, }
$$

where $D_{0}(\omega, k)$ is a function which determines the dispersion relation $\omega=\omega_{0}(k)$ when the dissipation is absent and the dissipation is responsible for the second term $D_{1}(\omega, k)$ the absolute value of which is much smaller than that of the first term. For plasma waves, the term $D_{0}(\omega, k)$ is determined by the Hermitian part of the dielectric tensor and the term $D_{1}(\omega, k)$ depends on both Hermitian and antiHermitian parts of the tensor (see, e.g., Ref. 27). Equation (B4) can be solved by means of a perturbation method. At the first step, the dissipation is neglected, and from the equa- tion $D_{0}(\omega, k)=0$ we obtain an approximate dispersion relation $\omega=\omega_{0}(k)$ giving the real part $\omega_{0}$ of the wave frequency,

$$
\omega=\omega_{0}+i \gamma,
$$

where $\gamma$ is the increment. Substituting Eq. (B5) into Eq. (B4), in the next approximation the increment is obtained,

$$
\gamma=-\left.\frac{D_{1}}{\partial D_{0} / \partial \omega}\right|_{\left(\omega_{0}(k), k\right)} .
$$

If medium is in a stable state as we suppose to be the case far ahead and far behind the shock, all disturbances should damp, $\gamma<0$. Hence,

$$
\left.\frac{D_{1}}{\partial D_{0} / \partial \omega}\right|_{\left(\omega_{0}(k), k\right)}>0 .
$$

Suppose that in the reference frame, where the unperturbed plasma is at rest, the shock wave is moving along the $x$ axis in the positive direction. Then in the shock frame both the precursor and wake are stationary, $\omega=0$, and the velocity of the medium upstream and downstream of the shock is negative, $U_{1,2}<0$. Using the formulas for the nonrelativistic Doppler effect, we find that in the reference frame, where the medium upstream or downstream is at rest, the precursor and wake waves have real frequencies,

$$
\omega=k U_{1,2},
$$

respectively, but the corresponding wave numbers will be complex, $k=k_{0}+i \kappa$, where $\kappa$ is an imaginary part of the wave number. Substituting Eq. (B7) into the dispersion relation (B4) and making use of the technique utilized above when finding the increment, we obtain

$$
\kappa=\left.\frac{D_{1}}{U_{1,2} \frac{\partial D_{0}}{\partial \omega}-\frac{\partial D_{0}}{\partial k}}\right|_{\left(k_{0} U_{1,2}, k_{0}\right)} .
$$

Using Eq. (B6) and the definition of the group velocity, this relationship can be rewritten as

$$
\kappa=\left.\frac{\gamma}{v_{\mathrm{gr}}-v_{\mathrm{ph}}}\right|_{\left(k_{0} U_{1,2}, k_{0}\right)} .
$$

If the group velocity exceeds the velocity of the flow, we see that $\kappa>0$ and the amplitude of the waves will vanish as $x$ $\rightarrow+\infty$ as should be the case for the precursor. For the wake wave trains, which vanish as $x \rightarrow-\infty$, the opposite condition should hold. The criteria (B2) are thereby proved.

Finally, it is worth noting that the well-known criteria for existence of the precursor and wake wave trains in the vicinity of a dispersive shock are quite universal and can be considered as necessary and sufficient conditions that turn out to be independent on both the nature of the waves and dissipative effects provided the medium far ahead and far behind the shock is stable and the dissipation is weak enough for weakly damping waves to exist. 


\section{APPENDIX C: NONLINEAR WHISTLER WAVES IN A PLASMA WITH HOT ELECTRONS}

Oblique nonlinear whistler waves in a cold plasma were studied for the first time by Kazantsev. ${ }^{49}$ If ions are cold and electrons are "warm" and isothermal, the similar problem was solved by Kakutani et al. ${ }^{50}$ In this appendix, we extend these results for electrons with an adiabatic equation of state.

For one-dimensional flows, the two-fluid MHD equations can be written as follows:

$\frac{\partial n}{\partial t}+\frac{\partial}{\partial x}\left(n V_{x}\right)=0$

$\frac{\partial V_{x}}{\partial t}+V_{x} \frac{\partial V_{x}}{\partial x}=\frac{e}{\left(m_{e}+m_{i}\right) c}\left[\left(V_{i y}-V_{e y}\right) B_{z}-\left(V_{i z}-V_{e z}\right) B_{y}\right]$

$$
-\frac{1}{n\left(m_{e}+m_{i}\right)} \frac{\partial p}{\partial x},
$$

$\frac{\partial \widetilde{V}_{i}}{\partial t}+V_{x} \frac{\partial \widetilde{V}_{i}}{\partial x}=\frac{e}{m_{i}} \widetilde{E}+i \frac{e}{m_{i} c}\left(V_{x} \widetilde{B}-\widetilde{V}_{i} B_{x}\right)$,

$\frac{\partial \widetilde{V}_{e}}{\partial t}+V_{x} \frac{\partial \widetilde{V}_{e}}{\partial x}=-\frac{e}{m_{e}} \widetilde{E}-i \frac{e}{m_{e} c}\left(V_{x} \widetilde{B}-\widetilde{V}_{e} B_{x}\right)$,

$\frac{1}{c} \frac{\partial \widetilde{E}}{\partial t}=i \frac{\partial \widetilde{B}}{\partial x}-\frac{4 \pi n e}{c}\left(\widetilde{V}_{i}-\widetilde{V}_{e}\right)$,

$\frac{1}{c} \frac{\partial \widetilde{B}}{\partial t}=-i \frac{\partial \widetilde{E}}{\partial x}$

$B_{x}=B_{0} \cos \theta=$ const,

$\frac{\partial p}{\partial t}+V_{x} \frac{\partial p}{\partial x}+\gamma p \frac{\partial V_{x}}{\partial x}=0$

where $\gamma$ is the specific heat ratio for electron component. We assume that quasineutrality holds and for both electrons and ions $n$ denote the number density. The $x$-components of the fluid velocity of electrons and ions are approximately equal and denoted by $V_{x}$. For brevity, we introduce the following complex parameters: transverse electric field $\widetilde{E}=E_{y}+i E_{z}$, transverse magnetic field $\widetilde{B}=B_{y}+i B_{z}$, and transverse fluid velocities $\widetilde{V}=V_{y}+i V_{z}$ of electron and ion components.

In addition to the continuity Eq. (C1), the system (C1)(C8) has the following conservation relations for momentum and energy:

$$
\begin{aligned}
& \frac{\partial}{\partial t}\left[n\left(m_{e}+m_{i}\right) V_{x}+\frac{1}{4 \pi c}\left(E_{y} B_{z}-E_{z} B_{y}\right)\right] \\
& +\frac{\partial}{\partial x}\left[n\left(m_{e}+m_{i}\right) V_{x}^{2}+p+\frac{1}{8 \pi}\left(\left|\widetilde{B}^{2}+\right| \widetilde{E}^{2}\right)\right]=0, \\
& \frac{\partial}{\partial t}\left[n\left(m_{e} \widetilde{V}_{e}+m_{i} \widetilde{V}_{i}\right)-\frac{i B_{x}}{4 \pi c} \widetilde{E}\right] \\
& \quad+\frac{\partial}{\partial x}\left[n V_{x}\left(m_{e} \widetilde{V}_{e}+m_{i} \widetilde{V}_{i}\right)-\frac{B_{x} \widetilde{B}}{4 \pi}\right]=0,
\end{aligned}
$$

$$
\begin{aligned}
& \frac{\partial}{\partial t}\left\{n\left[\left(m_{e}+m_{i}\right) \frac{V_{x}^{2}}{2}+m_{e} \frac{\left|\widetilde{V}_{e}\right|^{2}}{2}+m_{i} \frac{\left|\widetilde{V}_{i}\right|^{2}}{2}\right]\right. \\
& \left.+\frac{p}{\gamma-1}+\frac{|\widetilde{B}|^{2}+|\widetilde{E}|^{2}}{8 \pi}\right\} \\
& +\frac{\partial}{\partial x}\left\{n V_{x}\left[\left(m_{e}+m_{i}\right) \frac{V_{x}^{2}}{2}+m_{e} \frac{\left|\widetilde{V}_{e}\right|^{2}}{2}+m_{i} \frac{\left|\widetilde{V}_{i}\right|^{2}}{2}\right]\right. \\
& \left.+\frac{\gamma p V_{x}}{\gamma-1}+\frac{c}{4 \pi}\left(E_{y} B_{z}-E_{z} B_{y}\right)\right\}=0 .
\end{aligned}
$$

For a stationary solution, we can choose a reference frame such that the time derivatives vanish. Then from Maxwell equations it follows that $\widetilde{E}=$ const and we can let $E_{y}$ $=0$. Assume further that at infinity the plasma is undisturbed. Here we can let $\widetilde{V}_{e, i}=0$. Then electric and magnetic field components are

$$
\begin{aligned}
& B_{x}=B_{0} \cos \theta, \quad B_{y}=B_{0} \sin \theta, \quad B_{z}=0, \\
& E_{y} \equiv 0, \quad E_{z} \equiv-\frac{V_{x 0}}{c} B_{0} \sin \theta,
\end{aligned}
$$

respectively. For definiteness, in the following we assume that $\gamma=2$.

After some straightforward algebra we can easily obtain

$$
\begin{aligned}
& y^{2}+z^{2}=\sin ^{2} \theta+2 M_{A}^{2}(1-v)+\beta\left(1-\frac{1}{v^{2}}\right), \\
& y^{\prime \prime}+\Omega z^{\prime}+\cos ^{2} \theta(y-\sin \theta)-M_{A}^{2}(v y-\sin \theta)=0, \\
& z^{\prime \prime}-\Omega y^{\prime}+\left(\cos ^{2} \theta-v M_{A}^{2}\right) z=0,
\end{aligned}
$$

where $y=B_{y} / B_{0}$ and $z=B_{z} / B_{0}$ are the dimensionless magnetic field components, $M_{A}$ is the Alfvén Mach number, $\beta$ $=8 \pi p_{0} / B_{0}^{2}, \quad \Omega=\left(\mu^{1 / 2}+\mu^{-1 / 2}\right) \cos \theta, \quad$ and $\quad v \quad$ is the $x$-component of the dimensionless plasma velocity normalized to its unperturbed value at infinity. The primes denote the derivatives with respect to the dimensionless coordinate introduced by

$$
d \xi=\frac{e B_{0}}{\left(m_{e} m_{i}\right)^{1 / 2} v c} d x .
$$

At infinity we have $v=1, y=\sin \theta, z=0$, and first and second derivatives of the magnetic field vanish. Equations (C10)-(C11) can be written in the following form:

$$
\begin{aligned}
& y^{\prime \prime}+\Omega z^{\prime}+\frac{\partial \Psi}{\partial y}=0, \\
& z^{\prime \prime}-\Omega y^{\prime}+\frac{\partial \Psi}{\partial z}=0,
\end{aligned}
$$

where

$$
\Psi=\psi(r)+\left(M_{A}^{2}-\cos ^{2} \theta\right) y \sin \theta,
$$

and $r^{2}=y^{2}+z^{2}$. It is convenient to write the addend $\psi(r)$ in terms of the plasma velocity, 


$$
\begin{aligned}
\psi= & \frac{\beta \cos ^{2} \theta}{2}\left(1-\frac{1}{v^{2}}\right)+\beta M_{A}^{2}\left(\frac{1}{v}-1\right) \\
& +M_{A}^{2} \cos ^{2} \theta(1-v)+\frac{M_{A}^{4}}{2}\left(v^{2}-1\right) .
\end{aligned}
$$

These equations are similar to that describing the motion of a charged particle in a uniform magnetic field and nonuniform potential electric field and have an integral

$$
I_{1}=\frac{1}{2}\left(y^{\prime 2}+z^{\prime 2}\right)+\Psi(y, z)
$$

corresponding to the energy conservation of the particle.

For cold plasma $(\beta=0)$ the equations considered have approximate soliton-like solutions with a characteristic wavelength of several $c / \omega_{p e}$ for Mach number range ${ }^{49}$

$$
\frac{\cos ^{2} \theta}{4 \mu} \leqslant M_{A}^{2} \leqslant \frac{\cos ^{2} \theta}{2 \mu}
$$

(here and in the following we assume that $\cos ^{2} \theta \gg \mu$ ). The maximum amplitude of these waves, $|\cos \theta| / \mu^{1 / 2}$, is much greater than the undisturbed value of the magnetic field. We can use this fact to simplify the problem considered. If we neglect the second term on the right-hand side in Eq. (C14), it is easily seen that Eqs. (C12) and (C13) will have two "integrals of motion,"

$$
\begin{aligned}
& I_{1}=\frac{1}{2}\left(y^{\prime 2}+z^{\prime 2}\right)+\psi(r), \\
& I_{2}=r^{2}\left(\varphi^{\prime}-\frac{\Omega}{2}\right),
\end{aligned}
$$

where $\varphi=\arctan (z / y)$. Using these integrals, we can easily obtain

$$
\begin{aligned}
(\beta- & \left.M_{A}^{2} v^{3}\right)^{2} v^{\prime 2} \\
= & v^{2}(1-v)^{2}\left[2 M_{A}^{2} v^{2}-\beta(v+1)\right] \\
& \times\left[M_{A}^{4} v^{3}+M_{A}^{2}\left(M_{A}^{2}-2 M_{0}^{2}\right) v^{2}\right. \\
& \left.+\beta\left(M_{0}^{2}-2 M_{A}^{2}\right) v+\beta M_{0}^{2}\right],
\end{aligned}
$$

where $M_{0}^{2}=\cos ^{2} \theta+\Omega^{2} / 4 \approx \cos ^{2} \theta / 4 \mu$.

In the vicinity of the undisturbed state, where $v \approx 1$, Eq. (C17) takes the form

$$
v^{\prime 2} \approx 4(1-v)^{2}\left(M_{A}^{2}-M_{0}^{2}\right)
$$

We see that nontrivial solutions can exist only under the condition $M_{A} \geqslant M_{0}$.

At the top of the wave the last multiplier on the righthand side in Eq. (C17) vanishes,

$$
\begin{aligned}
& M_{A}^{4} v_{\text {top }}^{3}+M_{A}^{2}\left(M_{A}^{2}-2 M_{0}^{2}\right) v_{\text {top }}^{2}+\beta\left(M_{0}^{2}-2 M_{A}^{2}\right) v_{\text {top }} \\
& \quad+\beta M_{0}^{2}=0 .
\end{aligned}
$$

This equation is biquadratic with respect to $M_{A}$ and is easy to analyze. From Eq. (C18), we can obtain $M_{A}$-dependence of the plasma velocity at the top of the wave, $v_{\text {top }}$, find that these waves exist in the finite Mach number range

$$
M_{0} \leqslant M_{A} \leqslant M_{\text {nw }},
$$

and $v_{\text {top }}<1$, i.e., in the waves the plasma density is increased.

To determine $M_{\mathrm{nw}}$, we note at this boundary two zeros of Eq. (C18) coincide. Thereby we obtain the $\beta$-dependence of $M_{\text {nw }}$,

$$
M_{\mathrm{nw}}^{2}\left(\frac{2 M_{0}^{2}-M_{\mathrm{nw}}^{2}}{2 M_{\mathrm{nw}}^{2}-M_{0}^{2}}\right)^{3}-\beta=0 .
$$

In accordance with the results obtained by Kazantsev, ${ }^{49}$ we see that $M_{\mathrm{nw}}=2^{1 / 2} M_{0}$ for $\beta=0$. If $\beta$ is finite but not large, we have

$$
M_{\mathrm{nw}} \simeq 2^{1 / 2} M_{0}\left(1-\frac{3 \beta^{1 / 3}}{2^{7 / 3} M_{0}^{2 / 3}}\right) .
$$

We observe that for oblique waves such that $M_{0} \gg 1$, the thermal corrections can be small enough even for finite $\beta$ if $\beta \ll M_{0}^{2}$.

Now we can find the $\beta$-dependencies of the plasma velocity and magnetic field at the top of the wave for $M_{A}$ $=M_{\mathrm{nw}}$,

$$
\begin{gathered}
M_{0}^{2} \frac{v_{\text {top cr }}^{3}\left(v_{\text {top cr }}+2\right)}{1+2 v_{\text {top cr }}}-\beta=0, \\
r_{\text {top cr }}^{2}=\frac{27 M_{\mathrm{nw}}^{4}\left(M_{\mathrm{nw}}^{2}-M_{0}^{2}\right)^{2}}{\left(2 M_{\mathrm{nw}}^{2}-M_{0}^{2}\right)^{3}} .
\end{gathered}
$$

If $\beta$ is not too large, from the last equation and (C19) we get

$$
r_{\mathrm{top} \mathrm{cr}} \simeq 2 M_{0}\left(1-\frac{3 \beta^{1 / 3}}{2^{4 / 3} M_{0}^{2 / 3}}\right) .
$$

It is easily seen that the magnetic field at the top of the wave can considerably exceed its value far upstream of the wave not only in the cold plasma but for finite $\beta$ as well.

${ }^{1}$ R. Z. Sagdeev, in Reviews of Plasma Physics, edited by M. A. Leontovich (Consultants Bureau, New York, 1966), Vol. 4, p. 23.

${ }^{2}$ D. A. Tidman and N. A. Krall, Shock Waves in Collisionless Plasmas (Wiley-Interscience, New York, 1971).

${ }^{3}$ A. L. Velikovich and M. A. Liberman, Physics of Shock Waves in Gases and Plasmas (Nauka, Moscow, 1987) (in Russian).

${ }^{4}$ P. L. Auer, H. Hurwitz, Jr., and R. W. Kilb, Phys. Fluids 5, 298 (1962).

${ }^{5}$ J. W. M. Paul, G. C. Goldenbaum, A. Iiyoshi, L. S. Holmes, and R. A. Hardcastle, Nature (London) 216, 363 (1967).

${ }^{6}$ D. L. Morse, W. W. Destler, and P. L. Auer, Phys. Rev. Lett. 28, 13 (1972).

${ }^{7}$ O. Vaisberg, S. Klimov, G. Zastenker, M. Nozdratchev, A. Sokolov, V. Smirnov, S. Savin, and L. Avanov, Adv. Space Res. 4, 265 (1984).

${ }^{8}$ O. Vaisberg, G. Zastenker, V. Smirnov, Z. Němeček, J. Šafránková, L. Avanov, and E. Kolesnikova, Adv. Space Res. 6, 41 (1986).

${ }^{9}$ O. Vaisberg, G. Zastenker, V. Smirnov, Z. Němeček, J. Śafránková, L. Avanov, and E. Kolesnikova, Kosm. Issled. 24, 166 (1986) (in Russian).

${ }^{10}$ F. Bagenal, J. W. Belcher, E. C. Sittler, Jr., and R. P. Lepping, J. Geophys. Res., [Space Phys.] 92, 8603 (1987).

${ }^{11}$ A. A. Galeev, C. F. Kennel, V. V. Krasnoselskikh, and V. V. Lobzin, in Proceedings of the Joint Varenna-Abastumani International School and Workshop on Plasma Astrophysics, Varenna, Italy, 24 Aug.-3 Sept. 1988 (European Space Agency, Paris, 1988), ESA SP-285, Vol. 1, pp. 173-183.

${ }^{12} \mathrm{~V}$. V. Krasnoselskikh, V. V. Lobzin, and V. B. Rozov, in Proceedings of the Joint Varenna-Abastumani-ESA-Nagoya-Potsdam International Workshop on Plasma Astrophysics, Telavi, Georgia, 4-12 June 1990 (European Space Agency, Paris, 1990), ESA SP-311, pp. 103-107.

${ }^{13}$ M. M. Leroy, C. C. Goodrich, D. Winske, C. S. Wu, and K. Papadopoulos, Geophys. Res. Lett. 8, 1269 (1981). 
${ }^{14}$ M. M. Leroy, D. Winske, C. C. Goodrich, C. S. Wu, and K. Papadopoulos, J. Geophys. Res., [Space Phys.] 87, 5081 (1982).

${ }^{15}$ K. B. Quest, Phys. Rev. Lett. 54, 1872 (1985).

${ }^{16}$ K. B. Quest, J. Geophys. Res., [Space Phys.] 91, 8805 (1986).

${ }^{17}$ C. F. Kennel, J. P. Edmiston, and T. Hada, in Collisionless Shocks in the Heliosphere: A Tutorial Review, edited by B. Tsurutani and R. Stone (AGU, Washington, 1985), Geophysical Monograph 34, p. 1.

${ }^{18}$ V. V. Krasnosel'skikh, Sov. Phys. JETP 62, 282 (1985).

${ }^{19}$ A. A. Galeev, V. V. Krasnoselskikh, and V. V. Lobzin, Sov. J. Plasma Phys. 14, 697 (1988).

${ }^{20}$ A. A. Galeev, C. F. Kennel, V. V. Krasnoselskikh, and V. V. Lobzin, in Ref. 11, Vol. 1, pp. 165-171.

${ }^{21}$ V. V. Krasnoselskikh, M. A. Balikhin, H. St. C. Alleyne, S. I. Klimov, W. A. C. MierJedrzejowicz, A. K. Pardaens, A. Petrukovich, D. J. Southwood,

T. Vinogradova, and L. J. C. Woolliscroft, Adv. Space Res. 11, 15 (1991).

${ }^{22}$ B. Lembége and J. M. Dawson, Phys. Fluids 30, 1767 (1987).

${ }^{23}$ B. Lembége and P. Savoini, Phys. Fluids B 4, 3533 (1992).

${ }^{24}$ C. S. Wu, D. Winske, Y. M. Zhou, S. T. Tsai, P. Rodriguez, M. Tanaka, K. Papadopoulos, K. Akimoto, C. S. Lin, M. M. Leroy, and C. C. Goodrich, Space Sci. Rev. 37, 63 (1984).

${ }^{25}$ W. A. Livesey, C. T. Russel, and C. F. Kennel, J. Geophys. Res., [Space Phys.] 89, 6824 (1984).

${ }^{26}$ J. D. Scudder, A. Mangeney, C. Lacombe, C. C. Harvey, T. L. Aggson, R. R. Anderson, J. T. Gosling, G. Paschmann, and C. T. Russel, J. Geophys. Res., [Space Phys.] 91, 11019 (1986).

${ }^{27}$ V. L. Ginzburg, The Propagation of Electromagnetic Waves in Plasmas, 2nd ed. (Pergamon, Oxford, 1970).

${ }^{28}$ V. V. Krasnosel'skikh, E. N. Kruchina, G. Thejappa, and A. S. Volokitin, Astron. Astrophys. 149, 323 (1985).

${ }^{29}$ A. I. Akhiezer, A. A. Akhiezer, R. V. Polovin, A. G. Sitenko, and K. N. Stepanov, Plasma Electrodynamics (Pergamon, Oxford, 1975), Vols. 1, 2.

${ }^{30}$ L. D. Landau and E. M. Lifshitz, Electrodynamics of Continuous Media, 2nd ed. (Pergamon, Oxford, 1984).

${ }^{31}$ B. L. Rozhdestvensky and N. N. Yanenko, Systems of Quasilinear Equations (Nauka, Moscow, 1968) (in Russian).
${ }^{32}$ V. I. Karpman, Non-Linear Waves in Dispersive Media (Pergamon, Oxford, 1975).

${ }^{33}$ T. Taniuti, Prog. Theor. Phys. Suppl. 55, 1 (1974).

${ }^{34}$ G. B. Whitham, Linear and Nonlinear Waves (Wiley-Interscience, New York, 1974).

${ }^{35}$ P. I. Naumkin and I. A. Shishmarev, Nonlinear Nonlocal Equations in the Theory of Waves, Transl. Math. Monographs (American Mathematical Society, Providence, 1994), Vol. 133.

${ }^{36}$ P. I. Naumkin and I. A. Shishmarev, Dokl. Akad. Nauk SSSR 288, 90 (1986) (in Russian); English translation in Sov. Phys. Dokl. 31, 384 (1986).

${ }^{37}$ R. L. Seliger, Proc. R. Soc. London, Ser. A 303, 493 (1968).

${ }^{38}$ S. A. Gabov, Dokl. Akad. Nauk SSSR 246, 1292 (1979) (in Russian); English translation in Soviet Math. Dokl. 20 (1979).

${ }^{39}$ I. S. Gradshtein, Tables of Integrals, Series, and Products (Academic, New York, 1980).

${ }^{40}$ V. I. Karpman and R. Z. Sagdeev, Sov. Phys. Tech. Phys. 8, 606 (1964).

${ }^{41}$ V. I. Karpman, Space Sci. Rev. 16, 361 (1974).

${ }^{42}$ A. B. Langdon and B. F. Lasinski, in Methods of Computational Physics, edited by B. Alder et al. (Academic, New York, 1976), Vol. 16, p. 300.

${ }^{43}$ A. T. Lin, J. M. Sawson, and H. Okuda, Phys. Fluids 17, 1995 (1974).

${ }^{44}$ C. K. Birdsal, A. B. Langdon, and H. Okuda, in Methods of Computational Physics, edited by B. Alder et al. (Academic, New York, 1970), Vol. 9, p. 241.

${ }^{45}$ M. Balikhin, S. Walker, T. Dudok de Wit, H. Alleyne, L. Woolliscroft, W. Meir-Jedrzejowicz, and W. Baumjohann, Adv. Space Res. 20, 729 (1997).

${ }^{46}$ D. A. Tidman and T. G. Northrop, J. Geophys. Res. 73, 1543 (1968).

${ }^{47}$ J. K. Perez and T. G. Northrop, J. Geophys. Res. 75, 6011 (1970).

${ }^{48}$ F. V. Coroniti, J. Plasma Phys. 4, 265 (1970).

${ }^{49}$ A. P. Kazantsev, Zh. Éksp. Teor. Fiz. 44, 1283 (1963) (in Russian).

${ }^{50}$ T. Kakutani, T. Kawahara, and T. Taniuti, J. Phys. Soc. Jpn. 23, 1138 (1967). 